\title{
An Integrated Approach to Global Synchronization and State Estimation for Nonlinear Singularly Perturbed Complex Networks
}

\author{
Chenxiao Cai, Zidong Wang, Jing Xu, Xiaohui Liu and Fuad E. Alsaadi
}

\begin{abstract}
This paper aims to establish a unified framework to handle both the exponential synchronization and state estimation problems for a class of nonlinear singularly perturbed complex networks (SPCNs). Each node in the SPCN comprises both "slow" and "fast" dynamics that reflects the singular perturbation behavior. General sector-like nonlinear function is employed to describe the nonlinearities existing in the network. All nodes in the SPCN have the same structures and properties. By utilizing a novel Lyapunov functional and the Kronecker product, it is shown that the addressed SPCN is synchronized if certain matrix inequalities are feasible. The state estimation problem is then studied for the same complex network, where the purpose is to design a state estimator to estimate the network states through available output measurements such that dynamics (both "slow" and "fast") of the estimation error is guaranteed to be globally asymptotically stable. Again, a matrix inequality approach is developed for the state estimation problem. Two numerical examples are presented to verify the effectiveness and merits of the proposed synchronization scheme and state estimation formulation. It is worth mentioning that our main results are still valid even if the "slow" subsystems within the network are unstable.
\end{abstract}

Index Terms-Complex network, exponential synchronization, Kronecker product, singularly perturbed system, state estimation

\section{INTRODUCTION}

The past few decades have witnessed a surge of research outputs on the dynamics analysis of complex networks due primarily to their pervasive applications in a variety of physical systems and engineering plants [8], [10], [12], [18], [19], [30], [40] such as the internet, neural networks and genetic networks, etc. An increasing research interest has been devoted to the synchronization and stabilization problems for dynamical complex networks with each node representing a dynamical system [12], [32], [38]. The synchronization phenomenon has

This work was supported in part by the National Natural Science Foundation of China under Grants 61104064, 61174038, 61134009 and 61329301, the Fundamental Research Funds for Central Universities of China under Grant 30920140112005, the Deanship of Scientific Research (DSR) at King Abdulaziz University in Saudi Arabia under Grant 16-135-35-HiCi, the Royal Society of the U.K., and the Alexander von Humboldt Foundation of Germany.

C. Cai and J. Xu are with the School of Automation, Nanjing University of Science and Technology, Nanjing 210094, China. (Email: ccx5281@vip.163.com)

Z. Wang and X. Liu are with the Department of Computer Science, Brunel University, Uxbridge, Middlesex, UB8 3PH, United Kingdom. They are also with the Faculty of Engineering, King Abdulaziz University, Jeddah 21589 , Saudi Arabia. (Email: Zidong.Wang@brunel.ac.uk)

F. E. Alsaadi is with the Communication Systems and Networks (CSN) Research Group, Department of Electrical and Computer Engineering, Faculty of Engineering, King Abdulaziz University, Jeddah 21589, Saudi Arabia. proven to be of major concern to excite the collective behavior of complex dynamical networks [24], [25], and a rich body of literature has been available so far. For example, a sufficient condition for global synchronization and stability analysis has been derived in [19], [38], [39] based on a reference state and Lyapunov stability theory. Using the Lyapunov functional method and Kronecker product technique, the globally exponential synchronization and synchronizability have been studied in [27] for general dynamical networks. On the other hand, it is often the case that the complex network consists of a large number of nodes and only partial information about the network nodes is measureable through the network outputs. In such a case, the state estimation problem for complex networks based on available measurements becomes imperatively important and has stirred quite a lot of research attention, see [9], [25], [32], [33], [36] and the references therein. However, it is worth noting that, in almost all reported results, the node system in a complex network has been implicitly assumed to be regular, that is, the dynamics of the states of each node system evolves in the same time scale.

On the other hand, in practice, many dynamical systems possess two-time-scale characteristics, namely, an interaction of 'fast' and 'slow' dynamics such as aircraft and racket systems [34], electric power systems [1], [29] and biological systems [37]. Such kind of systems is governed by both fast and slow dynamics, and customarily referred to as the singularly perturbed systems (SPSs). In [34], a singularly perturbed structure has been assumed by artificial insertion of a small unit-valued parameter with highest derivative or some of the state variables of the nonlinear dynamical equations, where the four different structures of identifying the singular perturbation parameter have been presented in terms of the parameters of the nonlinear dynamical system. In [43], a differential geometric control approach has been provided to deal with the dynamics of the nodes of a power network modeled from the singular perturbation of the power flow equations. From a theoretical viewpoint, the singular perturbation provides us with a powerful tool to reduce the system order and separate the time scales of singularly perturbed systems [17], [23], but it also gives rise to significant difficulties in analyzing the system behaviors because of the small parasitic parameters multiplied by the time derivatives of the part of the system states.

In the context of complex networks, in the past decade, a variety of complex network models have been proposed and then thoroughly investigated. Examples include, but are not limited to, stochastic complex networks [20], [36], complex 
networks with imperfect measurements [33], [35], uncertain complex networks [21], [31] as well as complex networks with various kinds of transmission delays [9], [15]. It is noticeable that, for some complex networks such as power networks and neural networks, the node systems are often subject to singular perturbations. For example, in [43], the special singularly perturbed property of the classical distribution networks has been fully discussed that has led to a precise control with help from the singular perturbation approach, and the slow and the fast two-time-scale characteristics have been illustrated for a DC motor and a synchronous generator [7]. Furthermore, the neural network based control and observer design problems have been investigated in [22] for a class of singularly perturbed nonlinear systems with guaranteed $H_{\infty}$ control performance. In [4], the model predictive control problem has been handled for nonlinear singularly perturbed systems with application on a large-scale nonlinear reactorseparator process network which exhibits two-time-scale behavior. Unfortunately, a literature search reveals that little work has been devoted towards the dynamics analysis issue of the singularly perturbed complex networks especially when the singular perturbation phenomenon occurs on each node system. It is, therefore, the main focus of this paper to shorten such a gap by initiating a major study on the exponential synchronization and state estimation problems for a class of nonlinear singularly perturbed complex networks (SPCNs).

In this paper, we investigate the exponential synchronization and state estimation problems for a class of nonlinear SPCNS with each node subjecting to both 'slow' and 'fast' dynamics. All nodes in the SPCN are of the same structures and properties. Rather than the commonly used Lipschitz-type function, a more general sector-like nonlinear function is employed to describe the nonlinearities existing in the network. By utilizing a novel Lyapunov functional and the Kronecker product, the addressed synchronization problem is shown to be converted into the feasibility problem of a set of matrix inequalities. The subsequent state estimation problem is then dealt with for the same complex networks. Through available output measurements, a state estimator is designed to estimate the network states such that the dynamics of the estimation error is guaranteed to be globally asymptotically stable. Two simulation examples are provided to show the usefulness of the proposed global synchronization and estimation schemes. It is worth mentioning that our main results are still valid even if the 'slow subsystems within the network are unstable.

The main contributions of this paper are outlined as follows: 1) the exponential synchronization and state estimation problems are addressed, for the first time, for a class of general nonlinear SPCNs that allow directed and weighted topologies; 2) a unified framework is established for the addressed synchronization and problems for the addressed SPCNs exhibiting both the slow and fast dynamics; and 3) Sector-like nonlinearities enter into the system model and their impacts on the synchronization and estimation performances are analyzed. The rest of this paper is organized as follows. Problem formulation is presented and some preliminaries are introduced in Section II. In Section III, the globally exponential synchronization of the SPCNs is studied. Later, the state estimation of the SPCNs is discussed in Section IV where the state estimator is designed. In Section V, two numerical examples are given to demonstrate that our results are relevant to singularly perturbed dynamical networks. Finally, conclusions are drawn in Section VI.

Notations: The notations in this paper are standard. Throughout this paper, for real symmetric matrices $X$ and $Y$, the notation $X \leq Y$ (respectively, $X<Y$ ) means that the matrix $X-Y$ is negative semi-definite (respectively, negative definite). $I_{n}$ is the identity matrix of order $n . \mathbb{R}^{n}$ and $\mathbb{R}^{n \times m}$ denote the $n$-dimensional Euclidean space and the set of all real matrices with dimension $n \times m$, respectively. If $A$ is a square matrix, $\lambda_{\max }(A)$ (respectively, $\lambda_{\min }(A)$ ) means the largest (respectively, smallest) eigenvalue of $A$. The notation $A \otimes B$ stands for the Kronecker product of matrices $A$ and $B$. In symmetric block matrices, we use an asterisk "*" to represent a term that is induced by symmetry. The Hermitian part of a square matrix $M$ is denoted by $\operatorname{He}(M)=$ $M+M^{T}$. The superscript " $T$ " denotes matrix transposition and $\operatorname{diag}\{\cdots\}$ means a block-diagonal matrix. Matrices, if not explicitly stated, are assumed to have compatible dimensions for algebraic operations.

\section{Problem Formulation and Preliminaries}

Consider a nonlinear singularly perturbed complex network model consisting of $N$ linearly coupled identical nodes with full diagonal inner coupling, where each node is an $(n+m)$ dimensional dynamical singularly perturbed system given as follows:

$$
\left[\begin{array}{c}
\dot{x}_{i}(t) \\
\epsilon \dot{z}_{i}(t)
\end{array}\right]=f\left(x_{i}(t), z_{i}(t)\right)+\mathbf{I}(t)+c \sum_{j=1}^{N} d_{i j} \Theta\left[\begin{array}{c}
x_{j}(t) \\
z_{j}(t)
\end{array}\right] .
$$

Here, $i=1,2, \cdots, N . x_{i}(t)=\left[\begin{array}{llll}x_{1 i} & x_{2 i} & \cdots & x_{n i}\end{array}\right]^{T} \in$ $\mathbb{R}^{n}$ and $z_{i}(t)=\left[\begin{array}{llll}z_{1 i} & z_{2 i} & \cdots & z_{m i}\end{array}\right]^{T} \in \mathbb{R}^{m}$ are, respectively, the 'slow' and 'fast' state vectors of the $i$ th node. The constant $\epsilon(0<\epsilon \ll 1)$ is the singular perturbation parameter. $f\left(x_{i}(t), z_{i}(t)\right)=\left[\begin{array}{c}h\left(x_{i}(t)\right)+H z_{i}(t) \\ g\left(x_{i}(t)\right)+G z_{i}(t)\end{array}\right]$, where $h\left(x_{i}(t)\right) \in$ $\mathbb{R}^{n}$ and $g\left(x_{i}(t)\right) \in \mathbb{R}^{m}$ are continuously differentiable vectorvalued nonlinear functions, $H \in \mathbb{R}^{n \times m}$ and $G \in \mathbb{R}^{m \times m}$ are constant matrices. $\mathbf{I}(t)=\left[\begin{array}{c}\mathbf{I}_{n}(t) \\ \mathbf{I}_{m}(t)\end{array}\right]$ is an external input vector, where $\mathbf{I}_{n}(t)=\left[\begin{array}{llll}I_{1}(t) & I_{2}(t) & \cdots & I_{n}(t)\end{array}\right]_{T}^{T} \in$ $\mathbb{R}^{n}, \quad \mathbf{I}_{m}(t)=\left[\begin{array}{llll}I_{n+1}(t) & I_{n+2}(t) & \cdots & I_{n+m}(t)\end{array}\right]^{T} \in$ $\mathbb{R}^{m}$. Furthermore, $c>0$ is the coupling strength constant. $\Theta=\operatorname{diag}\{\Gamma, \Pi\}$ is a constant matrix linking the coupled variables with $\Gamma=\operatorname{diag}\left\{\gamma_{1}, \gamma_{2}, \cdots, \gamma_{n}\right\}$ and $\Pi=$ $\operatorname{diag}\left\{\pi_{1}, \pi_{2}, \cdots, \pi_{m}\right\}$, which implies that the $r$ th state variable of the $i$ th node of the network is only affected by the $r$ th state variables of other nodes of the network $(r=$ $1,2, \cdots, n, n+1, \cdots, n+m)$. The coupling matrix $D=$ $\left(d_{i j}\right)_{N \times N}$ is the Laplacian matrix representing the structure of the network, in which the off-diagonal elements $d_{i j}(i \neq j)$ are defined as follows:

$\left\{\begin{array}{c}d_{i j}=d_{j i}>0, \text { if the connection from node } j \text { to } i \text { exists } \\ d_{i j}=0, \text { otherwise }\end{array}\right.$ 
which means that the network topology could be directed and weighted. The diagonal elements of the matrix $D$ are determined by the following diffusive coupling conditions [42]:

$$
d_{i i}=-\sum_{j=1, j \neq i}^{N} d_{i j}, \quad i=1,2, \cdots, N
$$

Thus, the Laplacian matrix $D$ is a zero row-sum matrix.

For notational convenience, let us define the following notations:

$$
\begin{aligned}
& y_{i}(t)=\left[\begin{array}{l}
x_{i}(t) \\
z_{i}(t)
\end{array}\right], E_{\epsilon}=\left[\begin{array}{cc}
I_{n} & 0 \\
0 & \epsilon I_{m}
\end{array}\right], \\
& Y(t)=\left[\begin{array}{llll}
y_{1}^{T}(t) & y_{2}^{T}(t) & \cdots & y_{N}^{T}(t)
\end{array}\right]^{T}, \\
& F(Y(t))=\left[\begin{array}{llll}
f^{T}\left(y_{1}(t)\right) & f^{T}\left(y_{2}(t)\right) & \cdots & f^{T}\left(y_{N}(t)\right)
\end{array}\right]^{T}, \\
& \mathbb{I}(t)=\left[\begin{array}{llll}
\mathbf{I}^{T}(t) & \mathbf{I}^{T}(t) & \cdots & \mathbf{I}^{T}(t)
\end{array}\right]^{T}
\end{aligned}
$$

and then the network (1) can be rewritten in the following compact form by means of the Kronecker product:

$$
\left(I_{N} \otimes E_{\epsilon}\right) \dot{Y}(t)=F(Y(t))+\mathbb{I}(t)+c(D \otimes \Theta) Y(t) .
$$

The nonlinear vector-valued functions $h(\cdot)$ and $g(\cdot)$ are assumed to satisfy the following sector-like nonlinear functions which are more general than the traditional Lipschitz-type ones.

Assumption 1: [25], [26] The nonlinear functions $h(\cdot)$ and $g(\cdot)$ are continuous and satisfy

$$
\begin{aligned}
& \chi_{1}^{T} \chi_{2} \leq 0, \\
& {\psi_{1}}^{T} \psi_{2} \leq 0,
\end{aligned}
$$

for any $x_{i}(t), x_{j}(t) \in \mathbb{R}^{n}(i, j=1,2, \cdots, N)$, where

$$
\begin{aligned}
& \chi_{1}=h\left(x_{i}(t)\right)-h\left(x_{j}(t)\right)-B_{1}\left(x_{i}(t)-x_{j}(t)\right) \\
& \chi_{2}=h\left(x_{i}(t)\right)-h\left(x_{j}(t)\right)-B_{2}\left(x_{i}(t)-x_{j}(t)\right) \\
& \psi_{1}=g\left(x_{i}(t)\right)-g\left(x_{j}(t)\right)-D_{1}\left(x_{i}(t)-x_{j}(t)\right) \\
& \psi_{2}=g\left(x_{i}(t)\right)-g\left(x_{j}(t)\right)-D_{2}\left(x_{i}(t)-x_{j}(t)\right) .
\end{aligned}
$$

Here, $B_{1}, B_{2} \in \mathbb{R}^{n \times n}$ and $D_{1}, D_{2} \in \mathbb{R}^{m \times n}$ are constant matrices.

For the 'fast' part of the singularly perturbed complex network (1) or (3), as conventionally done in [11], we have the following assumption.

Assumption 2: The 'fast' subsystem of every node is stable, i.e. the state matrix $G$ is Hurwitz.

Furthermore, the following definition and lemma are provided for subsequent technical development of the paper.

Definition 1: The complex network (3) is said to be globally exponentially synchronized if, for any initial values $y_{i}(0)(i=1,2, \cdots, N)$, there exist constants $0<\epsilon^{*} \ll 1$, $\eta>0, T>0$ and $\beta>0$ such that

$$
\left\|y_{i}(t)-y_{j}(t)\right\| \leq \beta e^{-\eta t}
$$

holds for all $t>T$, any $\epsilon \in\left(0, \epsilon^{*}\right]$ and any $i, j=1,2, \cdots, N$.

Lemma 1: [25] Let $W=\left(w_{i j}\right)_{N \times N}, P \in \mathbb{R}^{n \times n}, x=$ $\left[\begin{array}{llll}x_{1}^{T} & x_{2}^{T} & \cdots & x_{N}^{T}\end{array}\right]^{T}$ and $y=\left[\begin{array}{llll}y_{1}^{T} & y_{2}^{T} & \cdots & y_{N}^{T}\end{array}\right]^{T}$ with $x_{i}, y_{i} \in \mathbb{R}^{n}(i=1,2, \cdots, N)$. If $W=W^{T}$ and each row sum of $W$ is zero, then

$$
x^{T}(W \otimes P) y=-\sum_{1 \leq i<j \leq N} w_{i j}\left(x_{i}-x_{j}\right)^{T} P\left(y_{i}-y_{j}\right) .
$$

In this paper, our main aim is to study the synchronization problem for the SPCN (3) by deriving sufficient conditions under which the network (3) is guaranteed to be exponential synchronized. Furthermore, we will extend the results obtained to design the desired state estimator for the same complex network model through available network measurements.

\section{SYNCHRONIZATION ANALYSIS}

In the section, the globally exponential synchronization problem for the SPCN (3) by the Lyapunov functional method.

Theorem 1: Let Assumption 1 and Assumption 2 hold. (i) For a given $\epsilon>0$, the network (3) can reach globally exponential synchronization if there exist two scalars $\delta_{1}>0$ and $\delta_{2}>0$, three positive definite matrices $P_{1} \in \mathbb{R}^{n \times n}$, $P_{3} \in \mathbb{R}^{m \times m}$ and $R \in \mathbb{R}^{m \times m}$, a matrix $P_{2} \in \mathbb{R}^{m \times n}$ such that the following linear matrix inequalities (LMIs) (8)-(9) hold:

$$
\begin{aligned}
E_{\epsilon} P_{\epsilon}= & E_{\epsilon}\left[\begin{array}{cc}
P_{1} & \epsilon P_{2}^{T} \\
P_{2} & P_{3}
\end{array}\right]>0 \\
\Omega(\epsilon, i, j)= & {\left[\begin{array}{ccccc}
\mathscr{A} & * & * & * & * \\
\mathscr{B}^{T} & \mathscr{D} & * & * & * \\
\mathscr{E}^{T} & 0 & -\delta_{1} I_{n} & * & * \\
\mathscr{F}^{T} & 0 & 0 & -\delta_{2} I_{m} & * \\
0 & 0 & \epsilon P_{2} & P_{3} & -R
\end{array}\right]<0, }
\end{aligned}
$$

where $1 \leq i<j \leq N$ and

$$
\begin{aligned}
\mathscr{A} & =c N d_{i j}\left(P_{1} \Gamma+\Gamma^{T} P_{1}\right)-\delta_{1} \tilde{B}_{1}-\delta_{2} \tilde{D}_{1}, \\
\mathscr{B} & =P_{1} H+P_{2}^{T} G+c N d_{i j}\left(P_{2}^{T} \Pi+\epsilon \Gamma^{T} P_{2}^{T}\right), \\
\mathscr{D} & =R+\mathbf{H e}\left(\epsilon P_{2} H+P_{3} G+c N d_{i j} P_{3} \Pi\right), \\
\mathscr{E} & =P_{1}+\delta_{1} \tilde{B}_{2}, \mathscr{F}=P_{2}^{T}+\delta_{2} \tilde{D}_{2}, \\
\tilde{B}_{1} & =\operatorname{He}\left(B_{1}^{T} B_{2}\right) / 2, \tilde{B}_{2}=\left(B_{1}^{T}+B_{2}^{T}\right) / 2, \\
\tilde{D}_{1} & =\operatorname{He}\left(D_{1}^{T} D_{2}\right) / 2, \tilde{D}_{2}=\left(D_{1}^{T}+D_{2}^{T}\right) / 2 .
\end{aligned}
$$

(ii) Let $P_{0}=\left.P_{\epsilon}\right|_{\epsilon=0}$ and $E_{0}=\left.E_{\epsilon}\right|_{\epsilon=0}$. If there exist two scalars $\delta_{1}>0$ and $\delta_{2}>0$, three positive definite matrices $P_{1} \in \mathbb{R}^{n \times n}, P_{3} \in \mathbb{R}^{m \times m}, R \in \mathbb{R}^{m \times m}$ and matrix $P_{2} \in$ $\mathbb{R}^{m \times n}$ such that

$$
\begin{aligned}
E_{0} P_{0} & =P_{0}^{T} E_{0} \geq 0 \\
\Omega(0, i, j) & =\left.\Omega(\epsilon, i, j)\right|_{\epsilon=0}<0,1 \leq i<j \leq N
\end{aligned}
$$

hold, then the network (3) can reach globally exponential synchronization for sufficiently small $\epsilon>0$.

Proof: (i) According to Assumption 1, it follows readily from (4) that

$\iota_{i j}^{T}(t)\left[\begin{array}{cc}\left(B_{1}^{T} B_{2}+B_{2}^{T} B_{1}\right) / 2 & -\left(B_{1}^{T}+B_{2}^{T}\right) / 2 \\ -\left(B_{1}+B_{2}\right) / 2 & I_{n}\end{array}\right] \iota_{i j}(t) \leq 0$

where $\iota_{i j}(t)=\left[\begin{array}{c}x_{i}(t)-x_{j}(t) \\ h\left(x_{i}(t)\right)-h\left(x_{j}(t)\right)\end{array}\right]$, or, equivalently, 


$$
\iota_{i j}^{T}(t)\left[\begin{array}{cc}
\tilde{B}_{1} & -\tilde{B}_{2} \\
-\tilde{B}_{2}^{T} & I_{n}
\end{array}\right] \iota_{i j}(t) \leq 0 .
$$

Similarly, we have from (5) that

$$
\varkappa_{i j}^{T}(t)\left[\begin{array}{cc}
\tilde{D}_{1} & -\tilde{D}_{2} \\
-\tilde{D}_{2}^{T} & I_{m}
\end{array}\right] \varkappa_{i j}(t) \leq 0,
$$

where $\varkappa_{i j}(t)=\left[\begin{array}{c}x_{i}(t)-x_{j}(t) \\ g\left(x_{i}(t)\right)-g\left(x_{j}(t)\right)\end{array}\right]$.

To deal with the synchronization of the network (3), we consider the following Lyapunov function:

$$
V(Y(t))=e^{\eta t} Y^{T}(t)\left(W \otimes E_{\epsilon} P_{\epsilon}\right) Y(t),
$$

where $W=\left[\begin{array}{cccc}N-1 & -1 & \cdots & -1 \\ -1 & N-1 & \cdots & -1 \\ \cdots & \cdots & \cdots & \cdots \\ -1 & -1 & \cdots & N-1\end{array}\right]_{N \times N}$ zero row-sum matrix. Then, the derivative of (14) along the trajectories of (3) is

$$
\begin{aligned}
\left.\dot{V}(Y(t))\right|_{(3)}= & \eta e^{\eta t} Y^{T}(t)\left(W \otimes E_{\epsilon} P_{\epsilon}\right) Y(t) \\
& +2 e^{\eta t} Y^{T}(t)\left(W \otimes P_{\epsilon}^{T}\right)\left(I_{N} \otimes E_{\epsilon}\right) \dot{Y}(t) \\
= & e^{\eta t}\left[Y^{T}(t)\left(W \otimes \eta E_{\epsilon} P_{\epsilon}\right) Y(t)\right. \\
& +2 Y^{T}(t)\left(W \otimes P_{\epsilon}^{T}\right)[F(Y(t)) \\
+ & \mathbb{I}(t)+c(D \otimes \Theta) Y(t)]]
\end{aligned}
$$

Referring to the structure of matrix $W$ and according to Lemma 1 , we can obtain that

$$
\begin{aligned}
\Lambda_{1}= & -\sum_{1 \leq i<j \leq N} w_{i j} \nu_{i j}^{T}(t) \eta E_{\epsilon} P_{\epsilon} \nu_{i j}(t) \\
= & \sum_{1 \leq i<j \leq N} \nu_{i j}^{T}(t) \eta E_{\epsilon} P_{\epsilon} \nu_{i j}(t), \\
\Lambda_{2}= & -2 \sum_{1 \leq i<j \leq N} w_{i j} \nu_{i j}^{T}(t) P_{\epsilon}^{T} \rho_{i j}(t) \\
= & 2 \sum_{1 \leq i<j \leq N} \nu_{i j}^{T}(t) P_{\epsilon}^{T} \rho_{i j}(t) \\
& +2 \sum_{1 \leq i<j \leq N} \nu_{i j}^{T}(t) P_{\epsilon}^{T}\left[\begin{array}{c}
H \\
G
\end{array}\right]\left(z_{i}(t)-z_{j}(t)\right)(17)
\end{aligned}
$$

where $\Lambda_{1}=Y^{T}(t)\left(W \otimes \eta E_{\epsilon} P_{\epsilon}\right) Y(t), \Lambda_{2}=2 Y^{T}(t)(W \otimes$ $\left.P_{\epsilon}^{T}\right) F(Y(t)), \nu_{i j}(t)=y_{i}(t)-y_{j}(t)=\left[\begin{array}{c}x_{i}(t)-x_{j}(t) \\ z_{i}(t)-z_{j}(t)\end{array}\right]$, $\rho_{i j}(t)=f\left(y_{i}(t)\right)-f\left(y_{j}(t)\right)=\left[\begin{array}{c}h\left(x_{i}(t)\right)-h\left(x_{j}(t)\right) \\ g\left(x_{i}(t)\right)-g\left(x_{j}(t)\right)\end{array}\right]$ and, consequently,

$$
\begin{aligned}
& 2 \nu_{i j}^{T}(t) P_{\epsilon}^{T} \rho_{i j}(t)=2\left(x_{i}(t)-x_{j}(t)\right)^{T}\left[\begin{array}{ll}
P_{1} & P_{2}^{T}
\end{array}\right] \rho_{i j}(t) \\
& \quad+2\left(z_{i}(t)-z_{j}(t)\right)^{T}\left[\begin{array}{ll}
\epsilon P_{2} & P_{3}
\end{array}\right] \rho_{i j}(t) .
\end{aligned}
$$

For any real vectors $a, b$ and any matrix $R>0$ of compatible dimensions, we have the following elementary inequality

$$
2 a^{T} b \leq a^{T} R^{-1} a+b^{T} R b
$$

and therefore

$$
2\left(z_{i}(t)-z_{j}(t)\right)^{T}\left[\begin{array}{ll}
\epsilon P_{2} & P_{3}
\end{array}\right] \rho_{i j}(t)
$$

$$
\begin{aligned}
& \leq\left(z_{i}(t)-z_{j}(t)\right)^{T} R\left(z_{i}(t)-z_{j}(t)\right) \\
& +\rho_{i j}^{T}(t)\left[\begin{array}{c}
\epsilon P_{2}^{T} \\
P_{3}
\end{array}\right] R^{-1}\left[\begin{array}{ll}
\epsilon P_{2} & P_{3}
\end{array}\right] \rho_{i j}(t) .
\end{aligned}
$$

For the term of the external disturbance $2 Y^{T}(t)(W \otimes$ $\left.P_{\epsilon}^{T}\right) \mathbb{I}(t) Y^{T}(t)$, one has

$$
\begin{aligned}
& \left(W \otimes P_{\epsilon}^{T}\right) \mathbb{I}(t)=\left[\begin{array}{ccc}
w_{11} P_{\epsilon}^{T} & \cdots & w_{1 N} P_{\epsilon}^{T} \\
\vdots & \ddots & \vdots \\
w_{N 1} P_{\epsilon}^{T} & \cdots & w_{N N} P_{\epsilon}^{T}
\end{array}\right]\left[\begin{array}{c}
\mathbf{I}(t) \\
\vdots \\
\mathbf{I}(t)
\end{array}\right] \\
& =\left[\begin{array}{c}
\sum_{j=1}^{N} w_{1 j} P_{\epsilon}^{T} \mathbf{I}(t) \\
\vdots \\
\sum_{j=1}^{N} w_{N j} P_{\epsilon}^{T} \mathbf{I}(t)
\end{array}\right]=\left[\begin{array}{c}
0 \\
\vdots \\
0
\end{array}\right] .
\end{aligned}
$$

Noting that the coupling matrix $D$ satisfies the diffusive coupling condition (2), it follows that $W D=N D$ and

$$
\left(W \otimes P_{\epsilon}^{T}\right)(c D \otimes \Theta)=c N D \otimes\left(P_{\epsilon}^{T} \Theta\right)
$$

which leads to

$$
\begin{aligned}
& 2 Y^{T}(t)\left(W \otimes P_{\epsilon}^{T}\right) c(D \otimes \Theta) Y(t) \\
& =2 Y^{T}(t)\left(c N D \otimes\left(P_{\epsilon}^{T} \Theta\right)\right) Y(t) \\
& =2 \sum_{1 \leq i<j \leq N} c N d_{i j} \nu_{i j}^{T}(t) P_{\epsilon}^{T} \Theta \nu_{i j}(t) .
\end{aligned}
$$

Substituting (16)-(23) into (15), it yields from (12) and (13) that

$$
\begin{aligned}
\left.\dot{V}(Y(t))\right|_{(3)} & \leq e^{\eta t} \sum_{1 \leq i<j \leq N}\left[\nu_{i j}^{T}(t) \eta E_{\epsilon} P_{\epsilon} \nu_{i j}(t)\right. \\
& +\left(x_{i}(t)-x_{j}(t)\right)^{T}\left[\begin{array}{c}
P_{1} \\
P_{2}
\end{array}\right]^{T} \rho_{i j}(t) \\
& +\rho_{i j}^{T}(t)\left[\begin{array}{c}
P_{1} \\
P_{2}
\end{array}\right]\left(x_{i}(t)-x_{j}(t)\right) \\
& +\rho_{i j}^{T}(t)\left[\begin{array}{c}
\epsilon P_{2}^{T} \\
P_{3}
\end{array}\right] R^{-1}\left[\begin{array}{ll}
\epsilon P_{2} & P_{3}
\end{array}\right] \rho_{i j}(t) \\
& +\nu_{i j}^{T}(t) P_{\epsilon}^{T}\left[\begin{array}{c}
H \\
G
\end{array}\right]\left(z_{i}(t)-z_{j}(t)\right) \\
& +\left(z_{i}(t)-z_{j}(t)\right)^{T}\left[H^{T} \quad G^{T}\right] P_{\epsilon} \nu_{i j}(t) \\
& +c N d_{i j} \nu_{i j}^{T}(t)\left(P_{\epsilon}^{T} \Theta+\Theta^{T} P_{\epsilon}\right) \nu_{i j}(t) \\
& +\left(z_{i}(t)-z_{j}(t)\right)^{T} R\left(z_{i}(t)-z_{j}(t)\right) \\
& \left.-\delta_{1} \iota_{i j}^{T}(t) M \iota_{i j}(t)-\delta_{2} \varkappa_{i j}^{T}(t) N \varkappa_{i j}(t)\right] \\
& =e^{\eta t} \sum_{1 \leq i<j \leq N} \zeta_{i j}^{T}(t) \Upsilon(\epsilon, i, j) \zeta_{i j}(t), \quad(24)
\end{aligned}
$$

where

$$
\begin{aligned}
& M=\left[\begin{array}{cc}
\tilde{B}_{1} & -\tilde{B}_{2} \\
-\tilde{B}_{2}^{T} & I_{n}
\end{array}\right], N=\left[\begin{array}{cc}
\tilde{D}_{1} & -\tilde{D}_{2} \\
-\tilde{D}_{2}^{T} & I_{m}
\end{array}\right], \\
& \zeta_{i j}(t)=\left[\begin{array}{c}
x_{i}(t)-x_{j}(t) \\
z_{i}(t)-z_{j}(t) \\
h\left(x_{i}(t)\right)-h\left(x_{j}(t)\right) \\
g\left(x_{i}(t)\right)-g\left(x_{j}(t)\right)
\end{array}\right] \\
& \Upsilon(\epsilon, i, j)=\left[\begin{array}{cccc}
\tilde{\mathscr{A}} & * & * & * \\
\tilde{\mathscr{B}}^{T} & \tilde{\mathscr{D}} & * & * \\
P_{1}+\delta_{1} \tilde{B}_{2}^{T} & 0 & \kappa_{1} & * \\
P_{2}+\delta_{2} \tilde{D}_{2}^{T} & 0 & \epsilon P_{3} R^{-1} P_{2} & \kappa_{2}
\end{array}\right]
\end{aligned}
$$




$$
\begin{aligned}
& \tilde{\mathscr{A}}=\eta P_{1}+c N d_{i j} \mathbf{H e}\left(P_{1} \Gamma\right)-\delta_{1} \tilde{B}_{1}-\delta_{2} \tilde{D}_{1}, \\
& \tilde{\mathscr{B}}=\epsilon \eta P_{2}^{T}+P_{1} H+P_{2}^{T} G+c N d_{i j}\left(P_{2}^{T} \Pi+\epsilon \Gamma^{T} P_{2}^{T}\right), \\
& \tilde{\mathscr{D}}=\epsilon \eta P_{3}+R+\mathbf{H e}\left(\epsilon P_{2} H+P_{3} G+c N d_{i j} P_{3} \Pi\right) . \\
& \kappa_{1}=\epsilon^{2} P_{2}^{T} R^{-1} P_{2}-\delta_{1} I_{n} \\
& \kappa_{2}=P_{3} R^{-1} P_{3}-\delta_{2} I_{m}
\end{aligned}
$$

Using Schur complement, $\Upsilon(\epsilon, i, j)<0$ is equivalent to

$$
\left[\begin{array}{ccccc}
\tilde{\mathscr{A}} & * & * & * & * \\
\tilde{\mathscr{B}}^{T} & \tilde{\mathscr{D}} & * & * & * \\
P_{1}+\delta_{1} \tilde{B}_{2}^{T} & 0 & -\delta_{1} I_{n} & * & * \\
P_{2}+\delta_{2} \tilde{D}_{2}^{T} & 0 & 0 & -\delta_{2} I_{m} & * \\
0 & 0 & \epsilon P_{2} & P_{3} & -R
\end{array}\right]<0
$$

According to (9), there exists a small constant $\eta>0$ such that (25) holds. Hence, the inequality (24) results in $\left.\dot{V}(Y(t))\right|_{(3)} \leq 0$, which implies $V(Y(t)) \leq V(Y(0))$. Therefore, $e^{\eta t} Y^{T}(t)\left(W \otimes E_{\epsilon} P_{\epsilon}\right) Y(t)$ is bounded and it can be obtained that

$$
\begin{aligned}
& w_{i j} \lambda_{\min }\left(E_{\epsilon} P_{\epsilon}\right)\left\|y_{i}(t)-y_{j}(t)\right\|^{2} \\
& \leq \sum_{1 \leq i<j \leq N} w_{i j}\left(y_{i}(t)-y_{j}(t)\right)^{T} E_{\epsilon} P_{\epsilon}\left(y_{i}(t)-y_{j}(t)\right) \\
& =Y^{T}(t)\left(W \otimes E_{\epsilon} P_{\epsilon}\right) Y(t)=O\left(e^{-\eta t}\right) .
\end{aligned}
$$

Hence, there exist constants $\eta>0$ and $\beta>0$ such that

$$
\left\|y_{i}(t)-y_{j}(t)\right\| \leq \beta e^{-\eta t} .
$$

According to Definition 1, it can be concluded that the globally exponential synchronization of the network (3) can be achieved under the condition of (9).

(ii) Similar to [11], [44], we can choose $P_{\epsilon}$ such that, for $\epsilon=0$, the functional $V(Y(t))$ makes sense with $E_{\epsilon}=E_{0}$ and $P_{\epsilon}=P_{0}$ in the descriptor case (i.e. $\epsilon=0$ in (3)). If the 'reduced-order' LMIs $\Omega(0, i, j)<0(1 \leq i<j \leq N)$ hold for some $\delta_{1}, \delta_{2}, P_{0}$ and $R$, then for sufficiently small $\epsilon$, the full-order LMIs (9) hold for the same $\delta_{1}, \delta_{2}, P_{1}, P_{2}, P_{3}$ and $R$. Then, in view of (i) in this theorem, the network (3) is globally exponential synchronized. This completes the proof.

Remark 1: In Theorem 1, the synchronization problem is studied for a new type of complex networks with singular perturbations, where the main result established involves all the information about on the system parameters including those reflecting the slow and fast dynamics as well as the nonlinearities. Due to the general nonlinearities introduced in the model, a quadratic Lyapunov function is used to derive the sufficient conditions that can be checked efficiently via the Matlab LMI Toolbox. It would be a possible topic of research to use non-quadratic Lyapunov functions or direct mathematical analysis techniques in order to reduce the unnecessary conservatism.

\section{State Estimation}

For the complex network (3), sometimes, we can only know the partial information about the states of the some network nodes from the network outputs. However, in order to make use of the networks in practice, it becomes necessary to estimate the node states through available network output. Suppose that the output vector of the $i$ th node of the network (3) is described by

$$
\mathscr{Y}_{i}(t)=C_{i} y_{i}(t)=\left[\begin{array}{cc}
C_{x i} & 0 \\
0 & C_{z i}
\end{array}\right]\left[\begin{array}{c}
x_{i}(t) \\
z_{i}(t)
\end{array}\right],
$$

where $i=1,2, \cdots, N, C_{x i} \in \mathbb{R}^{l_{1} \times n}$ and $C_{z i} \in \mathbb{R}^{l_{2} \times m}\left(l_{1}+\right.$ $\left.l_{2}=l\right)$ are known constant matrices and $\mathscr{Y}_{i}(t) \in \mathbb{R}^{l}$ is the measurement output of the $i$ th node.

The state estimator is of the following form

$\left(I_{N} \otimes E_{\epsilon}\right) \dot{\hat{Y}}(t)=F(\hat{Y}(t))+\mathbb{I}(t)+c(D \otimes \Theta) \hat{Y}(t)+K(\mathscr{Y}(t)-C \hat{Y}(t))$, where $\mathscr{Y}(t)=\left[\begin{array}{lllll}\mathscr{Y}_{1}^{T}(t) & \mathscr{Y}_{2}^{T}(t) & \ldots & \mathscr{Y}_{N}^{T}(t)\end{array}\right]^{(29)}$, $C=\operatorname{diag}\left\{C_{1}, \quad C_{2}, \cdots, \quad C_{N}\right\}, \quad$ and $K=$ $\operatorname{diag}\left\{K_{1}, \quad K_{2}, \cdots, \quad K_{N}\right\}$ are filter parameters to be designed. Here, $K_{i}=\operatorname{diag}\left\{K_{x i}, \quad K_{z i}\right\}$ and $K_{x i} \in \mathbb{R}^{n \times l_{1}}, K_{z i} \in \mathbb{R}^{m \times l_{2}}(i=1,2, \cdots, N)$.

Let $e(t)=\left[\begin{array}{llll}e_{1}^{T}(t) & e_{2}^{T}(t) & \cdots & e_{N}^{T}(t)\end{array}\right]^{T} \triangleq \hat{Y}(t)-Y(t)$ with $e_{i}(t)=\hat{y}_{i}(t)-y_{i}(t)=\left[\begin{array}{c}\hat{x}_{i}(t)-x_{i}(t) \\ \hat{z}_{i}(t)-z_{i}(t)\end{array}\right] \triangleq\left[\begin{array}{c}e_{x i}(t) \\ e_{z i}(t)\end{array}\right]$ and $\hat{F}(e(t)) \triangleq F(\hat{Y}(t))-F(Y(t))$. Then, from (3) and (29), we obtain the following the state error dynamics:

$$
\left(I_{N} \otimes E_{\epsilon}\right) \dot{e}(t)=-K C e(t)+\hat{F}(e(t))+c(D \otimes \Theta) e(t) .
$$

For convenience of development in the sequel, let

$$
\begin{aligned}
\Lambda_{3} & =\hat{T} \operatorname{diag}\left\{I_{n}, \epsilon I_{m}, \cdots I_{n}, \epsilon I_{m}\right\} \hat{T}^{-1} \\
& =\operatorname{diag}\left\{I_{n}, \cdots I_{n}, \epsilon I_{m}, \cdots \epsilon I_{m}\right\},
\end{aligned}
$$

where $\hat{T}=T_{1} T_{2} \cdots T_{N}$ is a product of a series of rowswitching elementary matrices $T_{i} \in \mathbb{R}^{N(n+m) \times N(n+m)}(i=$ $1,2, \cdots, N), \Lambda_{3}=\hat{T}\left(I_{N} \otimes E_{\epsilon}\right) \hat{T}^{-1}$. According to the properties of the row-switching elementary transformation, one has $\hat{T}^{-1}=\hat{T}$. Hence, we have the following equivalent form of the system (30)

$$
\Lambda_{3} \dot{e}(t)=-\hat{T} K C e(t)+\hat{T} \hat{F}(Y(t))+c \hat{T}(D \otimes \Theta) e(t),
$$

i.e.

$$
\begin{aligned}
& {\left[\begin{array}{cc}
I_{N n} & 0 \\
0 & \epsilon I_{N m}
\end{array}\right]\left[\begin{array}{l}
\dot{e}_{x}(t) \\
\dot{e}_{z}(t)
\end{array}\right]} \\
& =\left[\begin{array}{cc}
\kappa_{3} & 0 \\
0 & \kappa_{4}
\end{array}\right]\left[\begin{array}{l}
e_{x}(t) \\
e_{z}(t)
\end{array}\right]+\left[\begin{array}{l}
\tilde{h}\left(e_{x}(t)\right)+\tilde{H} e_{z}(t) \\
\tilde{g}\left(e_{x}(t)\right)+\tilde{G} e_{z}(t)
\end{array}\right],
\end{aligned}
$$

where

$$
\begin{aligned}
& \kappa_{3}=-K_{x} C_{x}+c D \otimes \Gamma, \\
& \kappa_{4}=-K_{z} C_{z}+c D \otimes \Pi \text {, } \\
& e_{x}(t)=\left[\begin{array}{llll}
e_{x 1}^{T}(t) & e_{x 2}^{T}(t) & \cdots & e_{x N}^{T}(t)
\end{array}\right]^{T} \in \mathbb{R}^{N n}, \\
& e_{z}(t)=\left[\begin{array}{llll}
e_{z 1}^{T}(t) & e_{z 2}^{T}(t) & \cdots & e_{z N}^{T}(t)
\end{array}\right]^{T} \in \mathbb{R}^{N m}, \\
& K_{x} C_{x}=\operatorname{diag}\left\{K_{x 1} C_{x 1}, K_{x 2} C_{x 2}, \cdots, K_{x N} C_{x N}\right\} \text {, } \\
& K_{z} C_{z}=\operatorname{diag}\left\{K_{z 1} C_{z 1}, K_{z 2} C_{z 2}, \cdots, K_{z N} C_{z N}\right\} \text {, } \\
& \tilde{h}\left(e_{x}(t)\right)+\tilde{H} e_{z}(t)=\left[\begin{array}{c}
h\left(e_{x 1}(t)\right)+H e_{z 1}(t) \\
h\left(e_{x 2}(t)\right)+H e_{z 2}(t) \\
\cdots \\
h\left(e_{x N}(t)\right)+H e_{z N}(t)
\end{array}\right]
\end{aligned}
$$




$$
\begin{aligned}
& =\left[\begin{array}{c}
h\left(e_{x 1}(t)\right) \\
h\left(e_{x 2}(t)\right) \\
\cdots \\
h\left(e_{x N}(t)\right)
\end{array}\right]+\left(I_{N} \otimes H\right) e_{z}(t), \\
& \tilde{g}\left(e_{x}(t)\right)+\tilde{G} e_{z}(t)=\left[\begin{array}{c}
g\left(e_{x 1}(t)\right)+G e_{z 1}(t) \\
g\left(e_{x 2}(t)\right)+G e_{z 2}(t) \\
\cdots \\
g\left(e_{x N}(t)\right)+G e_{z N}(t)
\end{array}\right] \\
& =\left[\begin{array}{c}
g\left(e_{x 1}(t)\right) \\
g\left(e_{x 2}(t)\right) \\
\cdots \\
g\left(e_{x N}(t)\right)
\end{array}\right]+\left(I_{N} \otimes G\right) e_{z}(t) .
\end{aligned}
$$

The next goal is to choose a suitable $K_{i}$ such that $\hat{Y}(t)$ asymptotically approaches $Y(t)$. From Assumption 1, it is easy to verify that

$$
\begin{aligned}
& {\left[\begin{array}{c}
e_{x}(t) \\
\tilde{h}\left(e_{x}(t)\right)
\end{array}\right]^{T}\left[\begin{array}{cc}
\tilde{\mathbb{B}}_{1} & -\tilde{\mathbb{B}}_{2} \\
-\tilde{\mathbb{B}}_{2}^{T} & I_{N n}
\end{array}\right]\left[\begin{array}{c}
e_{x}(t) \\
\tilde{h}\left(e_{x}(t)\right)
\end{array}\right] \leq 0,} \\
& {\left[\begin{array}{c}
e_{x}(t) \\
\tilde{g}\left(e_{x}(t)\right)
\end{array}\right]^{T}\left[\begin{array}{cc}
\tilde{\mathbb{D}}_{1} & -\tilde{\mathbb{D}}_{2} \\
-\tilde{\mathbb{D}}_{2}^{T} & I_{N m}
\end{array}\right]\left[\begin{array}{c}
e_{x}(t) \\
\tilde{g}\left(e_{x}(t)\right)
\end{array}\right] \leq 0,}
\end{aligned}
$$

where

$$
\begin{aligned}
\tilde{\mathbb{B}}_{1} & =\operatorname{diag}\{\overbrace{\tilde{B}_{1}, \tilde{B}_{1}, \cdots, \tilde{B}_{1}}^{N}\}, \\
\tilde{\mathbb{B}}_{2} & =\operatorname{diag}\{\overbrace{\tilde{B}_{2}, \tilde{B}_{2}, \cdots, \tilde{B}_{2}}^{N}\}, \\
\tilde{\mathbb{D}}_{1} & =\operatorname{diag}\{\overbrace{\tilde{D}_{1}, \tilde{D}_{1}, \cdots, \tilde{D}_{1}}^{N}\}, \\
\tilde{\mathbb{D}}_{2} & =\operatorname{diag}\{\overbrace{\tilde{D}_{2}, \tilde{D}_{2}, \cdots, \tilde{D}_{2}}^{N}\} .
\end{aligned}
$$

For the error system (30) or (32), we have the following result.

Theorem 2: (i) Let Assumptions 1 and 2 hold. For given $\epsilon>0, K_{x}=\operatorname{diag}\left\{K_{x 1}, K_{x 2}, \cdots, K_{x N}\right\}$ and $K_{z}=$ $\operatorname{diag}\left\{K_{z 1}, K_{z 2}, \cdots, K_{z N}\right\}$, the error system (30) or (32) is globally asymptotically stable if there exist two scalars $\delta_{1}>0$ and $\delta_{2}>0$, matrix $\mathbf{P}_{\epsilon}=\left[\begin{array}{cc}\mathbf{P}_{1} & \epsilon \mathbf{P}_{2}^{T} \\ \mathbf{P}_{2} & \mathbf{P}_{3}\end{array}\right]$ with $\mathbf{P}_{1}=\operatorname{diag}\left\{P_{11}, P_{12}, \cdots, P_{1 N}\right\}>0, \mathbf{P}_{2}=$ $\operatorname{diag}\left\{P_{21}, P_{22}, \cdots, P_{2 N}\right\}, \mathbf{P}_{3}=\operatorname{diag}\left\{P_{31}, P_{32}, \cdots, P_{3 N}\right\}>$ $0, P_{1 i} \in \mathbb{R}^{n \times n}, \quad P_{2 i} \in \mathbb{R}^{m \times n}, P_{3 i} \in \mathbb{R}^{m \times m}(i=$ $1,2, \cdots, N)$ and matrix $Q=\operatorname{diag}\left\{Q_{1}, Q_{2}, \cdots, Q_{N}\right\}>0$ with $Q_{i} \in \mathbb{R}^{m \times m}(i=1,2, \cdots, N)$ such that the following LMIs hold:

$$
\begin{aligned}
& \mathbf{E}_{\epsilon} \mathbf{P}_{\epsilon}>0, \\
& \Delta(\epsilon)=\left[\begin{array}{ccccc}
\mathscr{E} & * & * & * & * \\
\mathscr{F} & \mathscr{G} & * & * & * \\
\mathbf{P}_{1}+\delta_{1} \tilde{\mathbb{B}}_{2}^{T} & 0 & -\delta_{1} I_{N n} & * & * \\
\mathbf{P}_{2}+\delta_{2} \tilde{\mathbb{D}}_{2}^{T} & 0 & * & -\delta_{2} I_{N m} & * \\
0 & 0 & \epsilon \mathbf{P}_{2} & \mathbf{P}_{3} & -Q
\end{array}\right]<0
\end{aligned}
$$

${ }^{(36)}$ Hence, it follows that

where

$$
\begin{aligned}
\mathbf{E}_{\epsilon} & =\operatorname{diag}\left\{I_{N n}, \epsilon I_{N m}\right\}, \\
\mathscr{E} & =\mathbf{H e}\left(-\mathbf{P}_{1} K_{x} C_{x}+c \mathbf{P}_{1}(D \otimes \Gamma)\right)-\delta_{1} \tilde{\mathbb{B}}_{1}-\delta_{2} \tilde{\mathbb{D}}_{1},
\end{aligned}
$$

$$
\begin{aligned}
\mathscr{F}= & -\epsilon \mathbf{P}_{2} K_{x} C_{x}-C_{z}^{T} K_{z}^{T} \mathbf{P}_{2}+c \epsilon \mathbf{P}_{2}(D \otimes \Gamma) \\
& +c(D \otimes \Pi)^{T} \mathbf{P}_{2}+\tilde{H}^{T} \mathbf{P}_{1}+\tilde{G}^{T} \mathbf{P}_{2}, \\
\mathscr{G}= & Q+\mathbf{H e}\left(-\mathbf{P}_{3} K_{z} C_{z}+c \mathbf{P}_{3}(D \otimes \Pi)+\epsilon \mathbf{P}_{2} \tilde{H}+\mathbf{P}_{3} \tilde{G}\right) .
\end{aligned}
$$

(ii) For given $K_{x}=\operatorname{diag}\left\{K_{x 1}, K_{x 2}, \cdots, K_{x N}\right\}$ and $K_{z}=\operatorname{diag}\left\{K_{z 1}, K_{z 2}, \cdots, K_{z N}\right\}$, the error system (30) or (32) is globally asymptotically stable for sufficiently small $\epsilon>0$ if there exist two scalars $\delta_{1}>0$ and $\delta_{2}>0$, matrix $\mathbf{P}_{0}=\left.\mathbf{P}_{\epsilon}\right|_{\epsilon=0}=$ $\left[\begin{array}{cc}\mathbf{P}_{1} & 0 \\ \mathbf{P}_{2} & \mathbf{P}_{3}\end{array}\right]$ with $\mathbf{P}_{1}^{T}=\mathbf{P}_{1}=\operatorname{diag}\left\{P_{11}, P_{12}, \cdots, P_{1 N}\right\}>$ $0, \quad \mathbf{P}_{2}=\operatorname{diag}\left\{P_{21}, P_{22}, \cdots, P_{2 N}\right\}, \quad \mathbf{P}_{3}^{T}=\mathbf{P}_{3}=$ $\operatorname{diag}\left\{P_{31}, P_{32}, \cdots, P_{3 N}\right\}>0, P_{1 i} \in \mathbb{R}^{n \times n}, P_{2 i} \in$ $\mathbb{R}^{m \times n}, P_{3 i} \in \mathbb{R}^{m \times m}(i=1,2, \cdots, N)$ and matrix $Q=$ $\operatorname{diag}\left\{Q_{1}, Q_{2}, \cdots, Q_{N}\right\}>0$ with $Q_{i} \in \mathbb{R}^{m \times m}(i=$ $1,2, \cdots, N)$ satisfying the following LMIs:

$$
\begin{aligned}
\mathbf{E}_{0} \mathbf{P}_{0} & =\mathbf{P}_{0}^{T} \mathbf{E}_{0} \geq 0, \\
\Delta(0) & =\left.\Delta(\epsilon)\right|_{\epsilon=0}<0,
\end{aligned}
$$

where $\mathbf{E}_{0}=\left.\mathbf{E}_{\epsilon}\right|_{\epsilon=0}$.

Proof: (i) Consider the following Lyapunov functional:

$$
V(e(t))=e^{T}(t) \mathbf{E}_{\epsilon} \mathbf{P}_{\epsilon} e(t),
$$

where $\mathbf{E}_{\epsilon}=\left[\begin{array}{cc}I_{N n} & 0 \\ 0 & \epsilon I_{N m}\end{array}\right], \mathbf{P}_{\epsilon}=\left[\begin{array}{cc}\mathbf{P}_{1} & \epsilon \mathbf{P}_{2}^{T} \\ \mathbf{P}_{2} & \mathbf{P}_{3}\end{array}\right], \mathbf{P}_{1}^{T}=$ $\mathbf{P}_{1}>0, \mathbf{P}_{3}^{T}=\mathbf{P}_{3}>0$. Differentiating (32) with respect to $t$ along the trajectories of (32), we obtain

$$
\begin{aligned}
\left.\dot{V}(e(t))\right|_{(32)}= & 2\left[\begin{array}{c}
e_{x}(t) \\
e_{z}(t)
\end{array}\right]^{T} \mathbf{P}_{\epsilon}^{T}\left(\left[\begin{array}{cc}
\kappa_{3} & 0 \\
0 & \kappa_{4}
\end{array}\right]\left[\begin{array}{l}
e_{x}(t) \\
e_{z}(t)
\end{array}\right]\right. \\
& \left.+\left[\begin{array}{c}
\tilde{h}\left(e_{x}(t)\right)+\tilde{H} e_{z}(t) \\
\tilde{g}\left(e_{x}(t)\right)+\tilde{G} e_{z}(t)
\end{array}\right]\right) .
\end{aligned}
$$

Due to (19), there exists a matrix $Q>0\left(Q \in \mathbb{R}^{N m \times N m}\right)$ such that

$$
\begin{aligned}
& 2\left[\begin{array}{l}
e_{x}(t) \\
e_{z}(t)
\end{array}\right]^{T} \mathbf{P}_{\epsilon}^{T}\left[\begin{array}{c}
\tilde{h}\left(e_{x}(t)\right)+\tilde{H} e_{z}(t) \\
\tilde{g}\left(e_{x}(t)\right)+\tilde{G} e_{z}(t)
\end{array}\right] \\
& =2 e_{x}^{T}(t)\left[\begin{array}{ll}
\mathbf{P}_{1} & \mathbf{P}_{2}^{T}
\end{array}\right]\left[\begin{array}{l}
\tilde{h}\left(e_{x}(t)\right) \\
\tilde{g}\left(e_{x}(t)\right)
\end{array}\right] \\
& +2 e_{z}^{T}(t)\left[\begin{array}{ll}
\epsilon \mathbf{P}_{2} & \mathbf{P}_{3}
\end{array}\right]\left[\begin{array}{l}
\tilde{h}\left(e_{x}(t)\right) \\
\tilde{g}\left(e_{x}(t)\right)
\end{array}\right] \\
& +2\left[\begin{array}{c}
e_{x}(t) \\
e_{z}(t)
\end{array}\right]^{T} \mathbf{P}_{\epsilon}^{T}\left[\begin{array}{c}
\tilde{H} \\
\tilde{G}
\end{array}\right] e_{z}(t) \\
& \leq 2 e_{x}^{T}(t)\left[\mathbf{P}_{1} \quad \mathbf{P}_{2}^{T}\right]\left[\begin{array}{l}
\tilde{h}\left(e_{x}(t)\right) \\
\tilde{g}\left(e_{x}(t)\right)
\end{array}\right]+e_{z}^{T}(t) Q e_{z}(t) \\
& +\left[\begin{array}{c}
\tilde{h}\left(e_{x}(t)\right) \\
\tilde{g}\left(e_{x}(t)\right)
\end{array}\right]^{T}\left[\begin{array}{c}
\epsilon \mathbf{P}_{2}^{T} \\
\mathbf{P}_{3}
\end{array}\right] Q^{-1}\left[\begin{array}{c}
\epsilon \\
\mathbf{P}_{3}^{T}
\end{array}\right]^{T}\left[\begin{array}{l}
\tilde{h}\left(e_{x}(t)\right) \\
\tilde{g}\left(e_{x}(t)\right)
\end{array}\right] \\
& +2\left[\begin{array}{c}
e_{x}(t) \\
e_{z}(t)
\end{array}\right]^{T} \mathbf{P}_{\epsilon}^{T}\left[\begin{array}{c}
\tilde{H} \\
\tilde{G}
\end{array}\right] e_{z}(t) .
\end{aligned}
$$

$$
\begin{aligned}
& \left.\dot{V}(e(t))\right|_{(32)} \\
& \leq 2\left[\begin{array}{c}
e_{x}(t) \\
e_{z}(t)
\end{array}\right]^{T} \mathbf{P}_{\epsilon}^{T}\left[\begin{array}{cc}
\kappa_{3} & 0 \\
0 & \kappa_{4}
\end{array}\right]\left[\begin{array}{l}
e_{x}(t) \\
e_{z}(t)
\end{array}\right]
\end{aligned}
$$




$$
\begin{aligned}
& +2 e_{x}^{T}(t)\left[\begin{array}{ll}
\mathbf{P}_{1} & \mathbf{P}_{2}^{T}
\end{array}\right]\left[\begin{array}{c}
\tilde{h}\left(e_{x}(t)\right) \\
\tilde{g}\left(e_{x}(t)\right)
\end{array}\right] \\
& +e_{z}^{T}(t) Q e_{z}(t) \\
& +\left[\begin{array}{c}
\tilde{h}\left(e_{x}(t)\right) \\
\tilde{g}\left(e_{x}(t)\right)
\end{array}\right]^{T}\left[\begin{array}{c}
\epsilon \mathbf{P}_{2}^{T} \\
\mathbf{P}_{3}
\end{array}\right] Q^{-1}\left[\begin{array}{c}
\epsilon \mathbf{P}_{2}^{T} \\
\mathbf{P}_{3}
\end{array}\right]^{T}\left[\begin{array}{c}
\tilde{h}\left(e_{x}(t)\right) \\
\tilde{g}\left(e_{x}(t)\right)
\end{array}\right] \\
& +2\left[\begin{array}{c}
e_{x}(t) \\
e_{z}(t)
\end{array}\right]^{T} \mathbf{P}_{\epsilon}^{T}\left[\begin{array}{c}
\tilde{H} \\
\tilde{G}
\end{array}\right] e_{z}(t) \\
& -\delta_{1}\left[\begin{array}{c}
e_{x}(t) \\
\tilde{h}\left(e_{x}(t)\right)
\end{array}\right]^{T}\left[\begin{array}{cc}
\tilde{\mathbb{B}}_{1} & -\tilde{\mathbb{B}}_{2} \\
-\tilde{\mathbb{B}}_{2}^{T} & I_{N n}
\end{array}\right]\left[\begin{array}{c}
e_{x}(t) \\
\tilde{h}\left(e_{x}(t)\right)
\end{array}\right] \\
& -\delta_{2}\left[\begin{array}{c}
e_{x}(t) \\
\tilde{g}\left(e_{x}(t)\right)
\end{array}\right]^{T}\left[\begin{array}{cc}
\tilde{\mathbb{D}}_{1} & -\tilde{\mathbb{D}}_{2} \\
-\tilde{\mathbb{B}}_{2}^{T} & I_{N m}
\end{array}\right]\left[\begin{array}{c}
e_{x}(t) \\
\tilde{g}\left(e_{x}(t)\right)
\end{array}\right] \\
& =\vartheta^{T} \mathcal{M} \vartheta
\end{aligned}
$$

where $\vartheta=\left[\begin{array}{llll}e_{x}^{T}(t) & e_{z}^{T}(t) & \tilde{h}^{T}\left(e_{x}(t)\right) & \tilde{g}^{T}\left(e_{x}(t)\right)\end{array}\right]^{T}$ and

$$
\mathcal{M}=\left[\begin{array}{cccc}
\mathscr{E} & \mathscr{F}^{T} & \mathbf{P}_{1}+\delta_{1} \tilde{\mathbb{B}}_{2} & \mathbf{P}_{2}^{T}+\delta_{2} \tilde{\mathbb{D}}_{2} \\
* & \mathscr{G} & 0 & 0 \\
* & * & \kappa_{5} & \epsilon \mathbf{P}_{2}^{T} Q^{-1} \mathbf{P}_{3} \\
* & * & * & \kappa_{6}
\end{array}\right]
$$

with $\kappa_{5}=-\delta_{1} I_{N n}+\epsilon^{2} \mathbf{P}_{2}^{T} Q^{-1} \mathbf{P}_{2}, \kappa_{6}=-\delta_{2} I_{N m}+\mathbf{P}_{3} Q^{-1} \mathbf{P}_{3}$. According to Schur Complement Lemma, $\mathcal{M}<0$ is equivalent to (35), which implies that $\left\|\hat{y}_{i}(t)-y_{i}(t)\right\| \rightarrow 0(t \rightarrow$ $\infty, i=1,2, \cdots, N)$.

(ii) Along the similar line as in the proof of (ii) of Theorem 1 , we can obtain a descriptor case when $\epsilon=0$. The 'reducedorder' results (37) and (38) hold naturally and the proof is therefore omitted.

From Theorem 2, it is still very difficult to find a global solution to the nonlinear inequality (36) in order to select an appropriate state estimator for system (3). Next, let us provide a procedure for constructing the state estimator for system (3). To this end, it follows from (19) that

$$
\begin{aligned}
& {\left[\begin{array}{cc}
0 & -\epsilon C_{x}^{T} K_{x}^{T} \mathbf{P}_{2}^{T}-\mathbf{P}_{2}^{T} K_{z} C_{z} \\
-\epsilon \mathbf{P}_{2} K_{x} C_{x}-C_{z}^{T} K_{z}^{T} \mathbf{P}_{2} & 0
\end{array}\right]} \\
& =\left[\begin{array}{cc}
0 & \epsilon \mathbf{P}_{2}^{T} \\
\mathbf{P}_{2} & 0
\end{array}\right]^{T}\left[\begin{array}{cc}
-K_{x} C_{x} & 0 \\
0 & -K_{z} C_{z}
\end{array}\right] \\
& +\left[\begin{array}{cc}
-K_{x} C_{x} & 0 \\
0 & -K_{z} C_{z}
\end{array}\right]^{T}\left[\begin{array}{cc}
0 & \epsilon \mathbf{P}_{2}^{T} \\
\mathbf{P}_{2} & 0
\end{array}\right] \\
& \leq\left[\begin{array}{cc}
0 & \epsilon \mathbf{P}_{2}^{T} \\
\mathbf{P}_{2} & 0
\end{array}\right]^{T}\left[\begin{array}{cc}
\mathbf{P}_{1}^{-1} & 0 \\
0 & \mathbf{P}_{3}^{-1}
\end{array}\right]\left[\begin{array}{cc}
0 & \epsilon \mathbf{P}_{2}^{T} \\
\mathbf{P}_{2} & 0
\end{array}\right] \\
& +\kappa_{7}^{T}\left[\begin{array}{cc}
\mathbf{P}_{1} & 0 \\
0 & \mathbf{P}_{3}
\end{array}\right] \kappa_{7}
\end{aligned}
$$

$$
\begin{aligned}
= & {\left[\begin{array}{cc}
0 & \epsilon \mathbf{P}_{2}^{T} \\
\mathbf{P}_{2} & 0
\end{array}\right]^{T}\left[\begin{array}{cc}
\mathbf{P}_{1}^{-1} & 0 \\
0 & \mathbf{P}_{3}^{-1}
\end{array}\right]\left[\begin{array}{cc}
0 & \epsilon \mathbf{P}_{2}^{T} \\
\mathbf{P}_{2} & 0
\end{array}\right] } \\
& +\kappa_{8}^{T}\left[\begin{array}{cc}
\mathbf{P}_{1}^{-1} & 0 \\
0 & \mathbf{P}_{3}^{-1}
\end{array}\right] \kappa_{8}
\end{aligned}
$$

where $\kappa_{7}=\operatorname{diag}\left\{-K_{x} C_{x},-K_{z} C_{z}\right\}$ and $\kappa_{8}=$ $\operatorname{diag}\left\{-\mathbf{P}_{1} K_{x} C_{x},-\mathbf{P}_{3} K_{z} C_{z}\right\}$.

Substituting (42) into (36) and using Schur Complement Lemma, the following results can be easily accessible from Theorem 2 and therefore the proof is omitted.

Theorem 3: (i) Let Assumption 1 and Assumption 2 hold. For a given $\epsilon>0$, if there exist two scalars $\delta_{1}>0$ and $\delta_{2}>0$ and matrices $\mathbf{P}_{\epsilon}=\left[\begin{array}{cc}\mathbf{P}_{1} & \epsilon \mathbf{P}_{2}^{T} \\ \mathbf{P}_{2} & \mathbf{P}_{3}\end{array}\right]$ with

$$
\begin{aligned}
& \mathbf{P}_{1}=\operatorname{diag}\left\{P_{11}, P_{12}, \cdots, P_{1 N}\right\}>0, \\
& \mathbf{P}_{2}=\operatorname{diag}\left\{P_{21}, P_{22}, \cdots, P_{2 N}\right\}, \\
& \mathbf{P}_{3}=\operatorname{diag}\left\{P_{31}, P_{32}, \cdots, P_{3 N}\right\}>0, \\
& Q=\operatorname{diag}\left\{Q_{1}, Q_{2}, \cdots, Q_{N}\right\}>0, \\
& \mathbf{Y}_{x}=\operatorname{diag}\left\{Y_{x 1}, Y_{x 2}, \cdots, Y_{x N}\right\}, \\
& \mathbf{Y}_{z}=\operatorname{diag}\left\{Y_{z 1}, Y_{z 2}, \cdots, Y_{z N}\right\}
\end{aligned}
$$

such that the following LMIs

$$
\begin{aligned}
\mathbf{E}_{\epsilon} \mathbf{P}_{\epsilon} & >0 \\
\Lambda(\epsilon) & <0
\end{aligned}
$$

hold, where

$$
\begin{aligned}
\tilde{\mathscr{E}} & =\mathbf{H e}\left(-\mathbf{Y}_{x} C_{x}+c \mathbf{P}_{1}(D \otimes \Gamma)\right)-\delta_{1} \tilde{\mathbb{B}}_{1}-\delta_{2} \tilde{\mathbb{D}}_{1}, \\
\tilde{\mathscr{F}} & =c \epsilon \mathbf{P}_{2}(D \otimes \Gamma)+c(D \otimes \Pi)^{T} \mathbf{P}_{2}+\tilde{H}^{T} \mathbf{P}_{1}+\tilde{G}^{T} \mathbf{P}_{2}, \\
\tilde{\mathscr{G}} & =Q+\mathbf{H e}\left(-\mathbf{Y}_{z} C_{z}+c \mathbf{P}_{3}(D \otimes \Pi)+\epsilon \mathbf{P}_{2} \tilde{H}+\mathbf{P}_{3} \tilde{G}\right),
\end{aligned}
$$

then the system (29) is a state estimator of the complex network (3). In this case, the estimator gain matrices can be chosen as $K_{x}=\mathbf{P}_{1}^{-1} \mathbf{Y}_{x}$ and $K_{z}=\mathbf{P}_{3}^{-1} \mathbf{Y}_{z}$.

(ii) Under Assumption 1, from the conclusion in (i), the system (29) becomes a state estimator of the complex network (3) for all sufficiently small $\epsilon>0$ if there exist two scalars $\delta_{1}>0, \delta_{2}>0$ and matrices $\mathbf{P}_{0}=\left.\mathbf{P}_{\epsilon}\right|_{\epsilon=0}, \mathbf{P}_{1}^{T}=\mathbf{P}_{1}=\operatorname{diag}\left\{P_{11}, P_{12}, \cdots, P_{1 N}\right\}>$ $0, \quad \mathbf{P}_{2}=\operatorname{diag}\left\{P_{21}, P_{22}, \cdots, P_{2 N}\right\}, \quad \mathbf{P}_{3}^{T}=\mathbf{P}_{3}=$ $\operatorname{diag}\left\{P_{31}, P_{32}, \cdots, P_{3 N}\right\}>0$ with $P_{1 i} \in \mathbb{R}^{n \times n}, P_{2 i} \in$ $\mathbb{R}^{m \times n}, \quad P_{3 i} \in \mathbb{R}^{m \times m}(i=1,2, \cdots, N)$, and $Q=$

$$
\Lambda(\varepsilon)=\left[\begin{array}{ccccccccc}
\tilde{\mathscr{E}} & * & * & * & * & * & * & * & * \\
\tilde{\mathscr{F}} & \tilde{\mathscr{G}} & * & * & * & * & * & * & * \\
\mathbf{P}_{1}+\delta_{1} \tilde{\mathbb{B}}_{2}^{T} & 0 & -\delta_{1} I_{N n} & * & * & * & * & * & * \\
\mathbf{P}_{2}+\delta_{2} \tilde{\mathbb{D}}_{2}^{T} & 0 & 0 & -\delta_{2} I_{N m} & * & * & * & * & * \\
0 & 0 & \epsilon \mathbf{P}_{2} & \mathbf{P}_{3} & -Q & * & * & * & * \\
-\mathbf{Y}_{x} C_{x} & 0 & 0 & 0 & 0 & -\mathbf{P}_{1} & * & * & * \\
0 & -\mathbf{Y}_{z} C_{z} & 0 & 0 & 0 & 0 & -\mathbf{P}_{3} & * & * \\
0 & \epsilon \mathbf{P}_{2}^{T} & 0 & 0 & 0 & 0 & 0 & -\mathbf{P}_{1} & * \\
\mathbf{P}_{2} & 0 & 0 & 0 & 0 & 0 & 0 & 0 & -\mathbf{P}_{3}
\end{array}\right]
$$


$\operatorname{diag}\left\{Q_{1}, Q_{2}, \cdots, Q_{N}\right\}>0$ with $Q_{i} \in \mathbb{R}^{m \times m} \quad(i=$ $1,2, \cdots, N)$ satisfying the following LMIs:

$$
\begin{aligned}
\mathbf{E}_{0} \mathbf{P}_{0} & =\mathbf{P}_{0}^{T} \mathbf{E}_{0} \geq 0, \\
\Lambda(0) & =\left.\Lambda(\epsilon)\right|_{\epsilon=0}<0
\end{aligned}
$$

where $\mathbf{E}_{0}=\left.\mathbf{E}_{\epsilon}\right|_{\epsilon=0}$. Under such conditions, the estimator gain matrices of the system (3) can be parameterized as $K_{x}=$ $\mathbf{P}_{1}^{-1} \mathbf{Y}_{x}$ and $K_{z}=\mathbf{P}_{3}^{-1} \mathbf{Y}_{z}$.

Remark 2: In Theorem 1 and Theorem 3, the exponential synchronization and the state estimation problems are thoroughly investigated for a class of general nonlinear SPCNs that allow directed and weighted topologies. Within a unified framework, the existence of the desired synchronization and estimation is guaranteed through solving a set of matrix inequalities, and both the slow and fast dynamics are handled using an integrated matrix analysis method. Especially, the sector-like nonlinearities in the system model do have a major impact on the synchronization and estimation performances as the sector bounds are explicitly reflected in the obtained existence conditions.

\section{NUMERICAL EXAMPLE}

In this section, two numerical examples are presented to illustrate the validity of the theoretical results on the synchronization and state estimation problems for the nonlinear SPCN (1). To better demonstrate the effectiveness of the criteria, two systems with different orders of the slow and fast states are chosen to verify the required performances.

Example 1: Consider the network model (1) with three nodes, where

$$
\begin{aligned}
& n=1, m=2, H=\left[\begin{array}{ll}
1 & 0.5
\end{array}\right], G=\left[\begin{array}{cc}
-3 & -3 \\
3 & -5
\end{array}\right], \\
& D=\left[\begin{array}{ccc}
-0.2 & 0.1 & 0.1 \\
0.1 & -0.2 & 0.1 \\
0.1 & 0.1 & -0.2
\end{array}\right], \Gamma=\left[\begin{array}{l}
-5
\end{array}\right], \\
& \Pi=\left[\begin{array}{cc}
-0.6 & 0 \\
0 & -0.5
\end{array}\right], c=0.2 .
\end{aligned}
$$

Let the nonlinear vector-valued functions be given by

$$
\begin{aligned}
& h\left(x_{1 i}(t)\right)=-2 x_{1 i}^{2}(t) e^{-0.01 x_{1 i}(t)}+5, \\
& g\left(x_{1 i}(t)\right)=\left[\begin{array}{c}
-x_{1 i}^{2}(t) e^{-0.03 x_{1 i}(t)}+5 \\
-x_{1 i}(t) e^{-0.03 x_{1 i}(t)}
\end{array}\right], i=1,2,3 .
\end{aligned}
$$

It can be verified that the following coefficients are satisfied Assumption 1.

$$
B_{1}=-4, B_{2}=-4, D_{1}=\left[\begin{array}{l}
-2 \\
-2
\end{array}\right], D_{2}=\left[\begin{array}{l}
-3 \\
-4
\end{array}\right] \text {. }
$$

When $\epsilon=0.05$, by using the Matlab LMI Toolbox, a feasible solution is found based on the LMI (9) as follows:

$$
\begin{aligned}
& P_{1}=3.3147, P_{2}=\left[\begin{array}{l}
1.2748 \\
0.3216
\end{array}\right], \\
& P_{3}=\left[\begin{array}{cc}
1.8713 & -0.2518 \\
-0.2518 & 1.5679
\end{array}\right], \\
& R=\left[\begin{array}{cc}
7.7185 & -0.7465 \\
-0.7465 & 8.0241
\end{array}\right],
\end{aligned}
$$

$$
\delta_{1}=0.8036, \delta_{2}=1.2475 .
$$

When $\epsilon=0$, the results are:

$$
\begin{aligned}
& P_{1}=3.2998, P_{2}=\left[\begin{array}{l}
1.2731 \\
0.3110
\end{array}\right], \\
& P_{3}=\left[\begin{array}{cc}
1.8325 & -0.2454 \\
-0.2454 & 1.5545
\end{array}\right], \\
& R=\left[\begin{array}{cc}
7.6175 & -0.7387 \\
-0.7387 & 7.9770
\end{array}\right] \\
& \delta_{1}=0.7918, \delta_{2}=1.2100
\end{aligned}
$$

Therefore, according to Theorem 1, we can conclude that the complex dynamical network (1) with given parameters is globally exponentially synchronized, which is further verified by the simulation result shown in Fig. 1. The figures display the synchronization behavior very well for all the states of network (1).

Next, let us validate the theoretical results for the state estimation problem. When $\epsilon=0.05$ and

$$
\begin{aligned}
& C_{x 1}=\left[\begin{array}{l}
0.9 \\
0.2
\end{array}\right], C_{x 2}=\left[\begin{array}{c}
1 \\
0.2
\end{array}\right], C_{x 3}=\left[\begin{array}{l}
0.1 \\
0.8
\end{array}\right], \\
& C_{z 1}=\left[\begin{array}{ll}
2 & 1
\end{array}\right], C_{z 2}=\left[\begin{array}{ll}
1 & 1
\end{array}\right], C_{z 3}=\left[\begin{array}{ll}
0.5 & 1
\end{array}\right],
\end{aligned}
$$

the estimator gain matrices are given by solving the LMIs (43) and (44) as

$$
K_{x}=\left[\begin{array}{cccccc}
0.1232 & 0.0390 & 0 & 0 & 0 & 0 \\
0 & 0 & 0.1704 & 0.0494 & 0 & 0 \\
0 & 0 & 0 & 0 & 0.2492 & 0.0214
\end{array}\right]
$$

and

$$
K_{z}=\left[\begin{array}{ccc}
0.2688 & 0 & 0 \\
0.1299 & 0 & 0 \\
0 & 0.3795 & 0 \\
0 & 0.1722 & 0 \\
0 & 0 & 0.5369 \\
0 & 0 & 0.1176
\end{array}\right] .
$$

When $\epsilon=0$, we get the following estimator gain matrices

$$
K_{x}=\left[\begin{array}{cccccc}
0.1193 & 0.0365 & 0 & 0 & 0 & 0 \\
0 & 0 & 0.1644 & 0.0459 & 0 & 0 \\
0 & 0 & 0 & 0 & 0.2398 & 0.0153
\end{array}\right]
$$

and

$$
K_{z}=\left[\begin{array}{ccc}
0.2733 & 0 & 0 \\
0.1274 & 0 & 0 \\
0 & 0.3840 & 0 \\
0 & 0.1683 & 0 \\
0 & 0 & 0.5445 \\
0 & 0 & 0.1033
\end{array}\right] .
$$

According to Theorem 3, the system (29) becomes a state estimator of the singularly perturbed complex network (1) or (3). The numerical simulation validates the theoretical results perfectly. Fig. 2 shows the evolutions of the states and their estimators of node 1, respectively. From the three figures, it is noticed that the state estimation approaches the original system state asymptotically. Specifically, the estimate errors are shown clearly in Figs. 3 for all states of node 1.

Example 2: Next, an complex network with unstable "slow" subsystems is selected to demonstrate that the proposed synchronization and estimation schemes still work well in such 
an unstable case. Similar to Example 1, the system parameters are chosen as follows:

$$
\begin{aligned}
& n=2, m=1, N=3, H=\left[\begin{array}{l}
3 \\
1
\end{array}\right], \\
& G=-2, \Gamma=\left[\begin{array}{cc}
1 & 0 \\
0 & -0.6
\end{array}\right], \Pi=-0.5, \\
& D=\left[\begin{array}{ccc}
-0.2 & 0.1 & 0.1 \\
0.1 & -0.2 & 0.1 \\
0.1 & 0.1 & -0.2
\end{array}\right], c=0.2, \\
& h(x(t))=\left[\begin{array}{cc}
2 x_{1 i}^{2}(t) e^{-0.01 x_{1 i}(t)}+5 \\
x_{2 i}^{2}(t) e^{-0.03 x_{2 i}(t)}+5
\end{array}\right], \\
& g(x(t))=\left[\begin{array}{c}
-x_{1 i}(t) e^{-0.03 x_{1 i}(t)}
\end{array}\right], i=1,2,3 .
\end{aligned}
$$

It can also be checked that

$$
\begin{aligned}
& B_{1}=\left[\begin{array}{cc}
-3 & 0.2 \\
0 & -4
\end{array}\right], B_{2}=\left[\begin{array}{cc}
-3 & 0.2 \\
0 & -2
\end{array}\right], \\
& D_{1}=\left[\begin{array}{ll}
1 & 0.2
\end{array}\right], D_{2}=\left[\begin{array}{ll}
0.8 & 0.2
\end{array}\right] .
\end{aligned}
$$

Solving LMIs (9), we have the following results. When $\epsilon=$ 0.2 ,

$$
\begin{aligned}
& P_{1}=\left[\begin{array}{cc}
3.4927 & -1.9848 \\
-1.9848 & 8.6963
\end{array}\right], \\
& P_{2}=\left[\begin{array}{ll}
-0.4450 & 0.1889
\end{array}\right], \\
& P_{3}=\left[\begin{array}{ll}
7.3937
\end{array}\right], \\
& R=[16.3262], \delta_{1}=3.0873, \delta_{2}=11.8949 .
\end{aligned}
$$

When $\epsilon=0$,

$$
\begin{aligned}
& P_{1}=\left[\begin{array}{cc}
0.7153 & -0.5602 \\
-0.5602 & 2.0382
\end{array}\right], \\
& P_{2}=\left[\begin{array}{ll}
-0.2516 & -0.0594
\end{array}\right], \\
& P_{3}=[2.1082], \\
& R=[4.5913], \delta_{1}=0.6737, \delta_{2}=3.5950 .
\end{aligned}
$$

According to Theorem 1, we can conclude that the complex dynamical network (1) is globally exponentially synchronized with given parameters. Also, the simulation result shown in Fig. 4 illustrates that all states of network (1) are synchronized even if the 'slow' subsystems are unstable. Next, let us discuss the state estimation case. When $\epsilon=0.2$ and

$$
\begin{aligned}
C_{x 1} & =\left[\begin{array}{ll}
0.9 & 0.3 \\
0.2 & 0.8
\end{array}\right], \\
C_{x 2} & =\left[\begin{array}{cc}
1 & 1 \\
0.2 & 0.7
\end{array}\right], \\
C_{x 3} & =\left[\begin{array}{ll}
0.1 & 0.7 \\
0.8 & 0.2
\end{array}\right], \\
C_{z 1} & =\left[\begin{array}{l}
2 \\
1
\end{array}\right], C_{z 2}=\left[\begin{array}{l}
1 \\
1
\end{array}\right], C_{z 3}=\left[\begin{array}{c}
0.5 \\
1
\end{array}\right],
\end{aligned}
$$

we solve (43) and (44) to obtain

$$
K_{x}=\left[\begin{array}{cccccc}
0.1384 & 0.1615 & 0 & 0 & 0 & 0 \\
0.1186 & 0.0045 & 0 & 0 & 0 & 0 \\
0 & 0 & 0.2420 & 0.0117 & 0 & 0 \\
0 & 0 & 0.0308 & 0.0684 & 0 & 0 \\
0 & 0 & 0 & 0 & 0.8594 & -0.1554 \\
0 & 0 & 0 & 0 & 0.0253 & 0.1036
\end{array}\right]
$$

and

$K_{z}=\left[\begin{array}{cccccc}0.0835 & 0.1226 & 0 & 0 & 0 & 0 \\ 0 & 0 & -0.0242 & 0.1708 & 0 & 0 \\ 0 & 0 & 0 & 0 & -0.0030 & 0.0816\end{array}\right]$.

When $\epsilon=0$, we get the following estimator gain matrices

$K_{x}=\left[\begin{array}{cccccc}0.1712 & 0.1642 & 0 & 0 & 0 & 0 \\ 0.1186 & 0.0535 & 0 & 0 & 0 & 0 \\ 0 & 0 & 0.2512 & 0.0736 & 0 & 0 \\ 0 & 0 & 0.0641 & 0.1025 & 0 & 0 \\ 0 & 0 & 0 & 0 & 0.8698 & -0.1519 \\ 0 & 0 & 0 & 0 & 0.0247 & 0.1081\end{array}\right]$

and

$K_{z}=\left[\begin{array}{cccccc}0.1226 & 0.1299 & 0 & 0 & 0 & 0 \\ 0 & 0 & 0.0401 & 0.1942 & 0 & 0 \\ 0 & 0 & 0 & 0 & 0.0415 & 0.1253\end{array}\right]$.

According to Theorem 3, the system (29) becomes a state estimator of the singularly perturbed complex network (1) or (3). Fig. 5 depicts the evolutions of the states and their estimators of node 1, which shows that the estimated state asymptotically tends to the original state. Furthermore, the estimation errors of states $x_{11}(t), x_{21}(t)$ and $z_{11}(t)$ are shown in Fig. 6.

\section{CONCLUSIONS}

In this paper, we have investigated the exponential synchronization and state estimation problems for a class of nonlinear SPCNS with each node subjecting to both 'slow' and 'fast' dynamics. By utilizing a novel Lyapunov functional and the Kronecker product, the addressed synchronization problem has been solved by checking the feasibility of a set of matrix inequalities. The subsequent state estimation problem has then been dealt with for the same complex networks. Through available output measurements, a state estimator has been designed to estimate the network states such that the dynamics of the estimation error is guaranteed to be globally asymptotically stable. Two simulation examples have been provided to show the usefulness of the proposed global synchronization and estimation schemes. Our main results are still valid even if the 'slow subsystems within the network are unstable.

It is worth mentioning that, in general, the slow dynamics could be either stable or unstable. Our main results for synchronization and state estimation problems are valid for both the stable and unstable cases, which has been confirmed through our two examples. On the other hand, in the derivation of our main results, we do need the technical assumption that that the fast dynamics is stable. In fact, if the fast dynamics is unstable, then the boundary layer caused by the fast dynamics doesn't decay and the corresponding synchronization/estimation problems cannot be dealt with by the unified approach since the fast and slow dynamics will operate on two distinctively different scales. We are currently developing more general techniques that would avoid the restrictive assumption and the results will appear in the near future. Also, it would be interesting to employ more up-to-date techniques (e.g. data-driven techniques [45], [46] and fuzzy control approaches [2], [3], [5], [14]) to enhance the practical relevance of the main results. 


\section{REFERENCES}

[1] E. Barany, S. Schaffer, K. Wedeward and S. Ball, Nonlinear controllability of singularly perturbed models of power flow networks, In: Proc. 43rd IEEE Conference on Decision and Control, Atlantis, Paradise Island, Bahamas, pp. 4826-4832, 2004.

[2] M. Chadli, A. Abdo and S. X. Ding, $H_{-} / H_{\infty}$ fault detection filter design for discrete-time Takagi-Sugeno fuzzy system, Automatica, vol. 49, no. 7, pp. 1996-2005, 2013.

[3] M. Chadli and H. R. Karimi, Robust Observer Design for Unknown Inputs Takagi-Sugeno Models, IEEE Trans. Fuzzy Systems, vol. 21, no. 1, pp. 158-164, 2013.

[4] X. Chen, M. Heidarinejad, J. Liu, D. M. de la Pena and P. D. Christofides, Model predictive control of nonlinear singularly perturbed systems: Application to a large-scale process network, J. Process Control, vol. 21, no. 9, pp. 1296-1305, 2011.

[5] Y. M. Chen, C.-M. Lin and C.-S. Hsueh, Emitter identification of electronic intelligence system using type-2 fuzzy classifier, Systems Science and Control Engineering, vol. 2, no. 1, pp. 389-397, 2014.

[6] J. H. Chow, Asymptotic stability of a class of non-linear singularly perturbed system, Journal of the Franklin Institute, vol. 305, no. 5, pp. 275-281, 1978.

[7] J. H. Chow and P. Kokotovic, Two-time-scale feedback design of a class of nonlinear systems, IEEE Transactions on Automatic Control, vol. 23, no. 3, pp. 438-443, 1978.

[8] C. Dangalchv, Satistical Mechanics and its Applications, Physica A, vol. 338, no. 3-4, pp. 659-671, 2004.

[9] D. Ding, Z. Wang, B. Shen and H. Shu, $H_{\infty}$ state estimation for discrete-time complex networks with randomly occurring sensor saturations and randomly varying sensor delays, IEEE Trans. Neural Networks and Learning Systems, vol. 23, no. 5, pp. 725-736, 2012.

[10] Z. Duan, G. Chen and L. Huang, Synchronization of weighted networks and complex synchronized regions, Physics Letters A, vol. 372, pp. 3741-3751, 2008.

[11] E. Fridman, Effects of small delays on stability of singularly perturbed systems, Automatica, vol. 38, pp. 897-902, 2002.

[12] H. Gao, J. Lam and G. Chen, New criteria for synchronization stability of general complex dynamical networks with coupling delays, Physics Letters A, vol. 360, pp. 263-273, 2006.

[13] R. Horn and C. Johnson, Matrix Analysis. Cambridge, U.K.: Cambridge Univ. Press, 1990.

[14] A. Iqbal, H. Abu-Rub and H. Nounou, Adaptive fuzzy logic-controlled surface mount permanent magnet synchronous motor drive, Systems Science and Control Engineering, vol. 2, no. 1, pp. 465-475, 2014.

[15] H. R. Karimi, Robust synchronization and fault detection of uncertain master-slave systems with mixed time-varying delays and nonlinear perturbations, Int. J. Control Automation and Systems, vol. 9, no. 4, pp. 671-680, 2011.

[16] H. Khalil, Feedback control of nonstandard singularly perturbed systems, IEEE Transactions on Automatic Control, vol. 34, pp. 1052-1060, 1989.

[17] P. Kokotovic, H. Khalil and J. O'Reilly, Singular perturbation methods in control: analysis and design, Academic Press, New York, 1986.

[18] G. Kumar and K. Kumar, Network security - an updated perspective, Systems Science and Control Engineering, vol. 2, no. 1, pp. 325-334, 2014.

[19] Z. Li and G. Chen, Global synchronization and asymptotic stability of complex dynamical networks, IEEE Transactions on Circuits and Systems II: Express briefs, vol. 53, no. 1, pp. 28-33, 2006.

[20] J. Liang, Z. Wang, Y. Liu and X. Liu, Global synchronization control of general delayed discrete-time networks with stochastic coupling and disturbances, IEEE Trans. Systems, Man and Cybernetics: Part B, vol. 38, no. 4, pp. 1073-1083, 2008.

[21] J. Liang, Z. Wang, X. Liu and P. Louvieris, Robust synchronization for 2-D discrete-time coupled dynamical networks, IEEE Trans. Neural Networks and Learning Systems, vol. 23, no. 6, pp. 942-953, 2012.

[22] K. -J. Lin, Stabilisation of singularly perturbed nonlinear systems via neural network-based control and observer design, Int. J. Systems Science, vol. 44, no. 10, pp. 1925-1933, 2013.

[23] H. Liu, F. Sun, K. He and Z. Sun, Survey of singularly perturbed control systems: theory and applications. Control Theory and Applications, vol. 20, no. 1, pp. 1-7, 2003.

[24] Y. Liu, Z. Wang and X. Liu, Exponential synchronization of complex networks with Markovian jump and mixed delays. Physics Letters A, vol. 372, pp. 3986-3998, 2008.

[25] Y. Liu, Z. Wang, J. Liang and X. Liu, Synchronization and state estimation for discrete-time complex networks with distributed delays. IEEE
Transactions on Systems, Manm and Cybernetics-Part B: Cybernetics, vol. 38,no. 5, pp. 1314-1325, 2008.

[26] Y. Liu, Z. Wang and X. Liu, Global exponential stability of generalized recurrent neural networks with discrete and distributed delays, Neural Netw, vol. 19, no. 5, pp. 667-675, 2006.

[27] J. Lu and D. W. C. Ho, Globally exponential synchronization and synchronizability for general dynamical networks, IEEE Transations on Systems, Man, and Cybernetics-Part B: Cybernetics, vol. 40, no. 2, pp. 350-361, 2010.

[28] W. Lu and T. Chen, New approach to synchronization analysis of linearly coupled ordinary differential systems, Phys. D, Nonlinear Phenom., vol. 213, no. 2, pp. 214-230, 2006.

[29] F. Ma and L. Fu, Principle of multi-time scale order reduction and its application in AC/DC hybrid power systems, In: Proc. International Conference on Electrical Machines and Systems, Wuhan, China, pp. 3951-3956, 2008.

[30] A. Mehrsai, H. R. Karimi and K.-D. Thoben, Integration of supply networks for customization with modularity in cloud and make-toupgrade strategy, Systems Science and Control Engineering, vol. 1, no. 1, pp. 28-42, 2013.

[31] M. Mostafa Asheghan and J. Miguez, Robust global synchronization of two complex dynamical networks, Chaos, vol. 23, no. 2, Art. no. 023108 , 2013.

[32] B. Shen, Z. Wang and X. Liu, Bounded $H_{\infty}$ synchronization and state estimation for discrete time-varying stochastic complex networks over a finite horizon, IEEE Transactions on Neural Networks, vol. 22, no. 1, pp. 145-157, 2011.

[33] B. Shen, Z. Wang, D. Ding and H. Shu, $H_{\infty}$ state estimation for complex networks with uncertain inner coupling and incomplete measurements, IEEE Trans. Neural Networks and Learning Systems, vol. 24, no. 12, pp. 2027-2037, 2013.

[34] D. Subbaram Naidu, Analysis of non-dimensional forms of singular perturbation structures for hypersonic vehicles, Acta Astronautica, vol. 66, pp. 577-586, 2010.

[35] Y. Tang, H. Gao, W. Zou and J. Kurths, Distributed synchronization in networks of agent systems with nonlinearities and random switchings, IEEE Trans. Cybernetics, vol. 43, no. 1, pp. 358-370, 2013.

[36] L. Wang, G. Wei and H. Shu, State estimation for complex networks with randomly occurring coupling delays, Neurocomputing, vol. 122, pp. 513-520, 2013.

[37] R. Wang, T. Zhou, Z. Jing and L. Chen, Modelling periodic oscillation of biological systems with multiple timescale networks, Systems Biology, vol. 1 , no. 1, pp. 71-84, 2004.

[38] X. Wang, Complex networks: topology, dynamics and synchronization, International Journal of Bifurcation and Chaos, vol. 12, no. 5, pp. 885916, 2002.

[39] X. Wang and G. Chen, Synchronization in scale-free dynamical networks: robustness and fragility, IEEE Transactions on Circuits and Systems. I, Fundamental Theory and Applications, vol. 49, no. 1, pp. 5462, 2002.

[40] Y. Wang and Z. Wang, A delay fractioning approach to global synchronization of delayed complex networks with stochastic disturbances, Physics Letters A, vol. 372, pp. 6066-6073, 2008.

[41] J. Winkelman, J. Chow, J. Allemong and P. V. Kokotovic, Multi-timescale analysis of a power system, Automatica, vol. 16, pp. 35-43, 1980.

[42] C. W. Wu and L. O. Chua, Synchronization in an array of linearly coupled dynamical systems, IEEE Transactions on Circuits and Systems. I, Fundamental Theory and Applications, vol. 42, no. 8, pp. 430-447, 1995.

[43] H. Xin, Z. Lu, Z. Qu, D. Gan and D. Qi, Cooperative control strategy for multiple photovoltaic generators in distribution networks, IET Control Theory and Applications, vol. 5, no. 14, pp. 1617-1629, 2011.

[44] H. Xu and K. Mizukami, Infinite-horizon differential games of singularly perturbed systems: a unified approach, Automatica, vol. 33, pp. 273-276, 1997.

[45] S. Yin, H. Luo and S. X. Ding, Real-time implementation of faulttolerant control systems with performance optimization, IEEE Trans. Industrial Electronics, vol. 61, no. 5, pp. 2402-2411, 2014.

[46] S. Yin, S. X. Ding, A. Haghani, H. Hao and P. Zhang, A comparison study of basic data-driven fault diagnosis and process monitoring methods on the benchmark Tennessee Eastman process, Journal of Process Control, vol. 22, no. 9, pp. 1567-1581, 2012. 

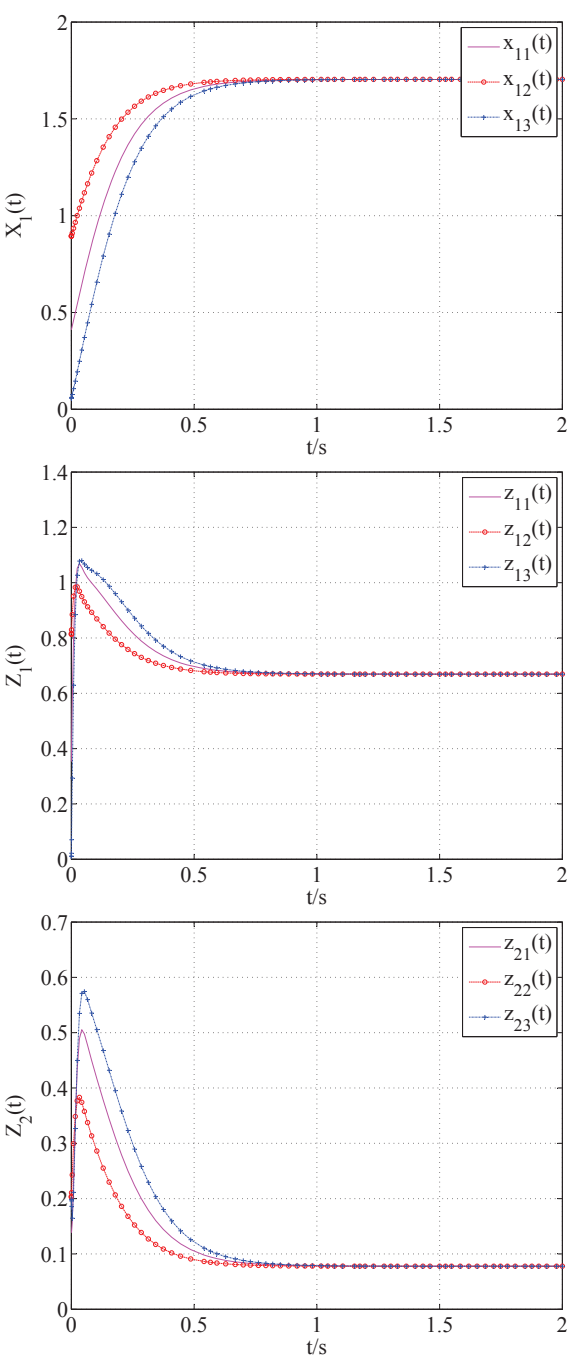

Fig. 1. Comparison of state trajectories of the states $X_{1}(t), Z_{1}(t)$ and $Z_{2}(t)$.

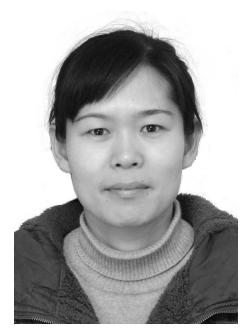

Chenxiao Cai received her Ph.D. degree in control theory and control engineering from Nanjing University of Science and Technology, Nanjing, China, in 2005. She is now an Associate Professor in the School of Automation, Nanjing University of Science and Technology, Nanjing, China. Her research interests cover singularly perturbed systems, complex networks and unmanned aerial vehicles.
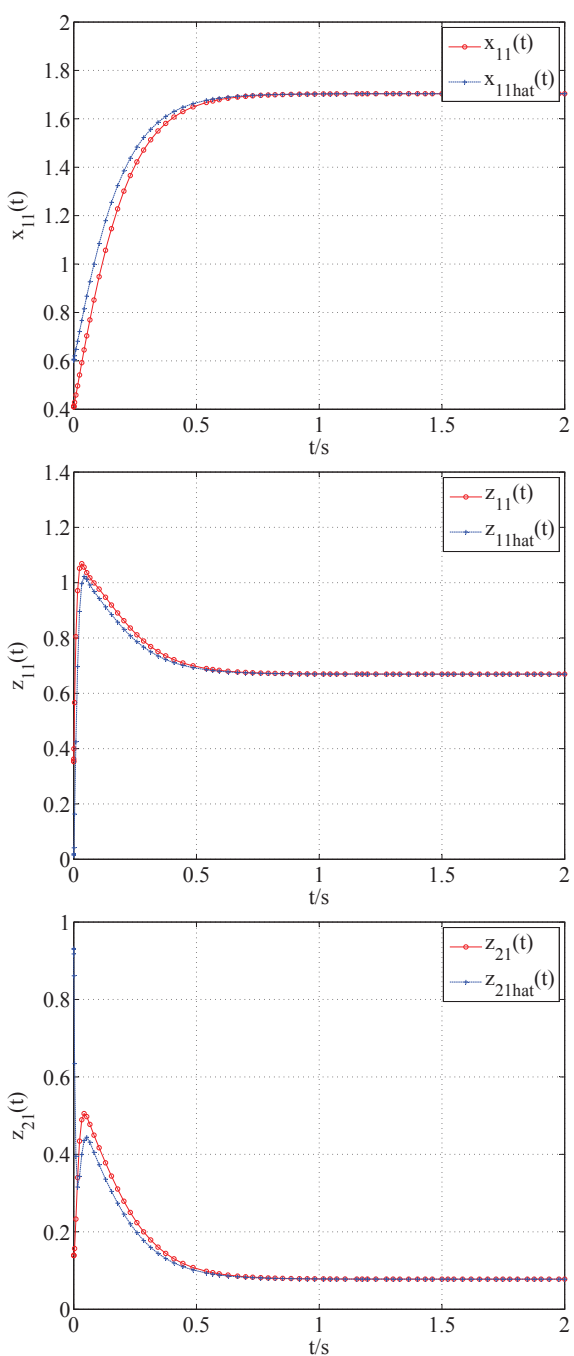

Fig. 2. State trajectories of the original states and their estimates of the first node system.

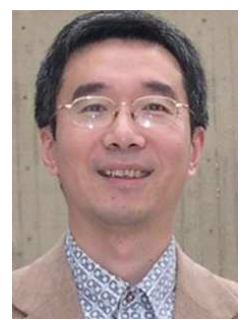

Zidong Wang (F'14) was born in Jiangsu, China, in 1966. He received the B.Sc. degree in mathematics in 1986 from Suzhou University, Suzhou, China, and the M.Sc. degree in applied mathematics in 1990 and the Ph.D. degree in electrical engineering in 1994, both from Nanjing University of Science and Technology, Nanjing, China.

$\mathrm{He}$ is currently Professor of Dynamical Systems and Computing in the Department of Information Systems and Computing, Brunel University, U.K. From 1990 to 2002, he held teaching and research appointments in universities in China, Germany and the UK. Prof. Wang's research interests include dynamical systems, signal processing, bioinformatics, control theory and applications. He has published more than 200 papers in refereed international journals. He is a holder of the Alexander von Humboldt Research Fellowship of Germany, the JSPS Research Fellowship of Japan, William Mong Visiting Research Fellowship of Hong Kong.

Prof. Wang serves as an Associate Editor for 12 international journals, including IEEE Transactions on Automatic Control, IEEE Transactions on Control Systems Technology, IEEE Transactions on Neural Networks, IEEE Transactions on Signal Processing, and IEEE Transactions on Systems, Man, and Cybernetics - Systems. He is a Fellow of the IEEE, a Fellow of the Royal Statistical Society and a member of program committee for many international conferences. 

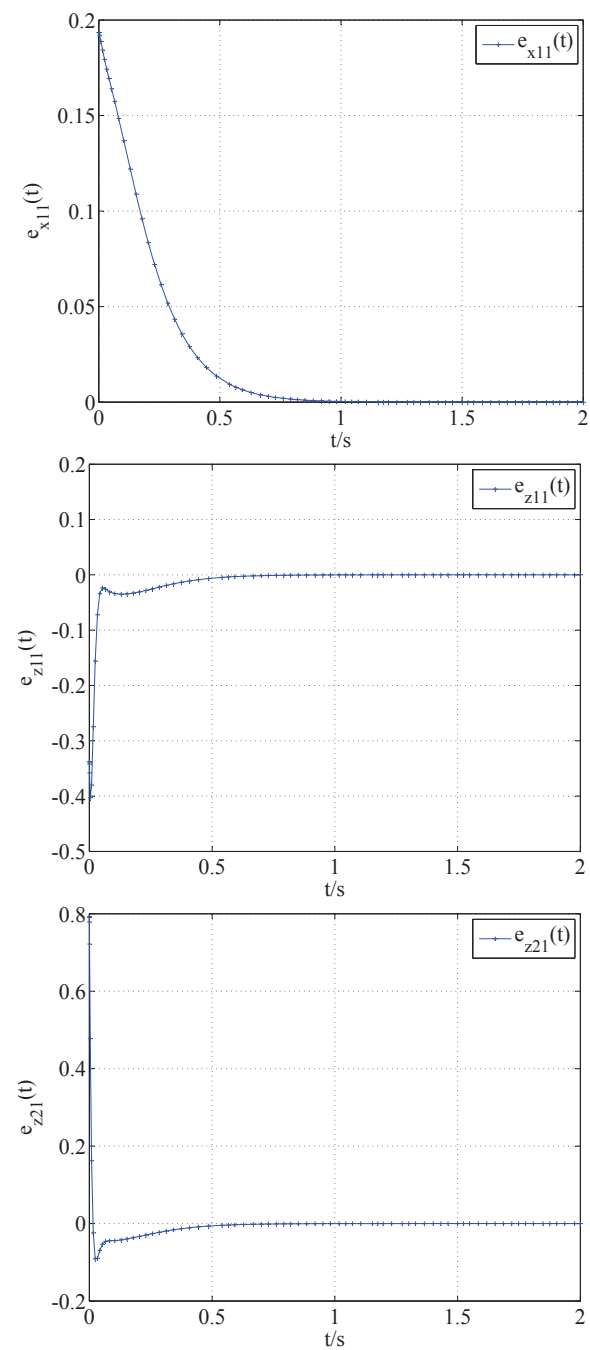

Fig. 3. Estimate error of the states of the first node system.

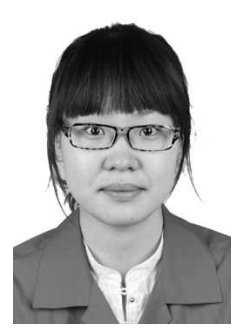

Jing $\mathrm{Xu}$ has been a Ph.D candidate in the School of Automation, Nanjing University of Science and Technology, Nanjing, China, since 2012. Her research interests includes singularly perturbed systsems, fault detection and unmanned aerial vehicle, etc.

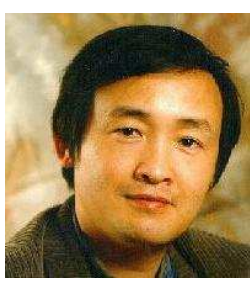

Xiaohui Liu received the B.Eng. degree in computing from Hohai University, Nanjing, China, in 1982 and the Ph.D. degree in computer science from Heriot-Watt University, Edinburg, U.K., in 1988.

$\mathrm{He}$ is currently a Professor of Computing at Brunel University. He leads the Intelligent Data Analysis (IDA) Group, performing interdisciplinary research involving artificial intelligence, dynamic systems, image and signal processing, and statistics, particularly for applications in biology, engineering and medicine. Professor Liu serves on editorial boards of four computing journals, founded the biennial international conference series on IDA in 1995, and has given numerous invited talks in bioinformatics, data mining and statistics conferences.
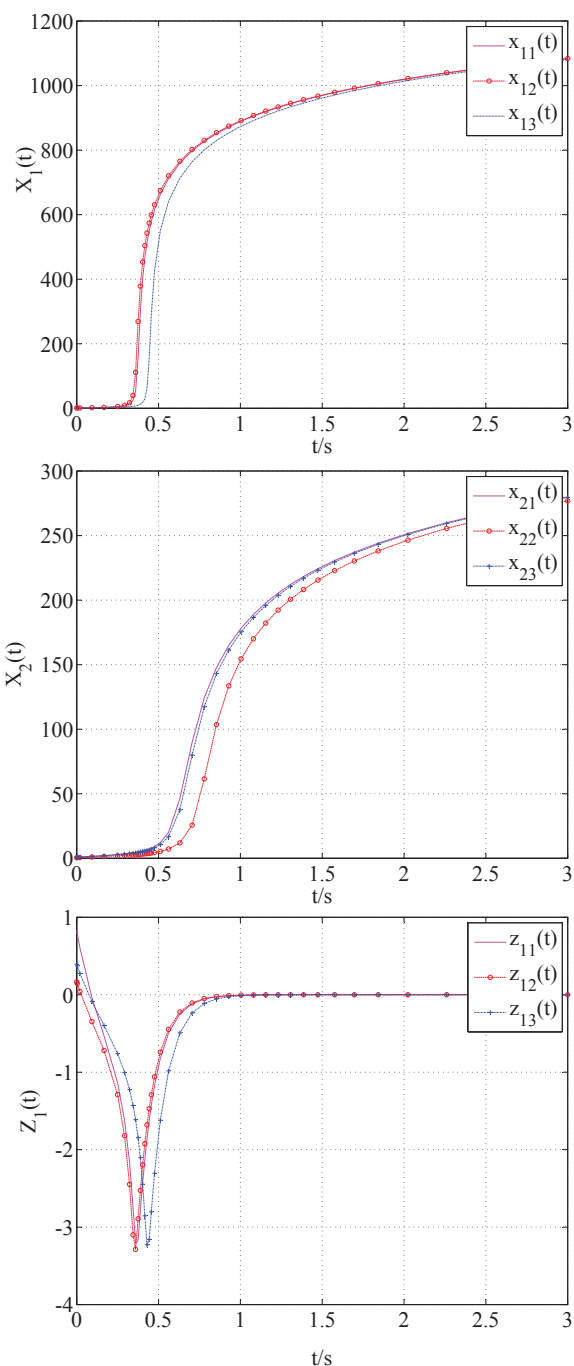

Fig. 4. Comparison of state trajectories of the states $X_{1}(t), X_{2}(t)$ and $Z_{1}(t)$.

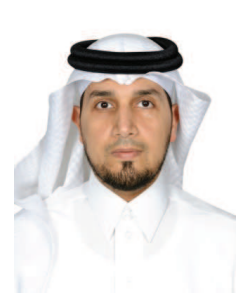

Fuad E. Alsaadi received the B.S. and M.Sc. degrees in electronic and communication from King AbdulAziz University, Jeddah, Saudi Arabia, in 1996 and 2002. He then received the Ph.D. degree in Optical Wireless Communication Systems from the University of Leeds, Leeds, UK, in 2011.

Between 1996 and 2005, he worked in Jeddah as a communication instructor in the College of Electronics \& Communication. He is currently an assistant professor of the Electrical and Computer Engineering Department within the Faculty of Engineering,

King Abdulaziz University, Jeddah, Saudi Arabia. He published widely in the top IEEE communications conferences and journals and has received the Carter award, University of Leeds for the best $\mathrm{PhD}$. He has research interests in optical systems and networks, signal processing, synchronization and systems design. 

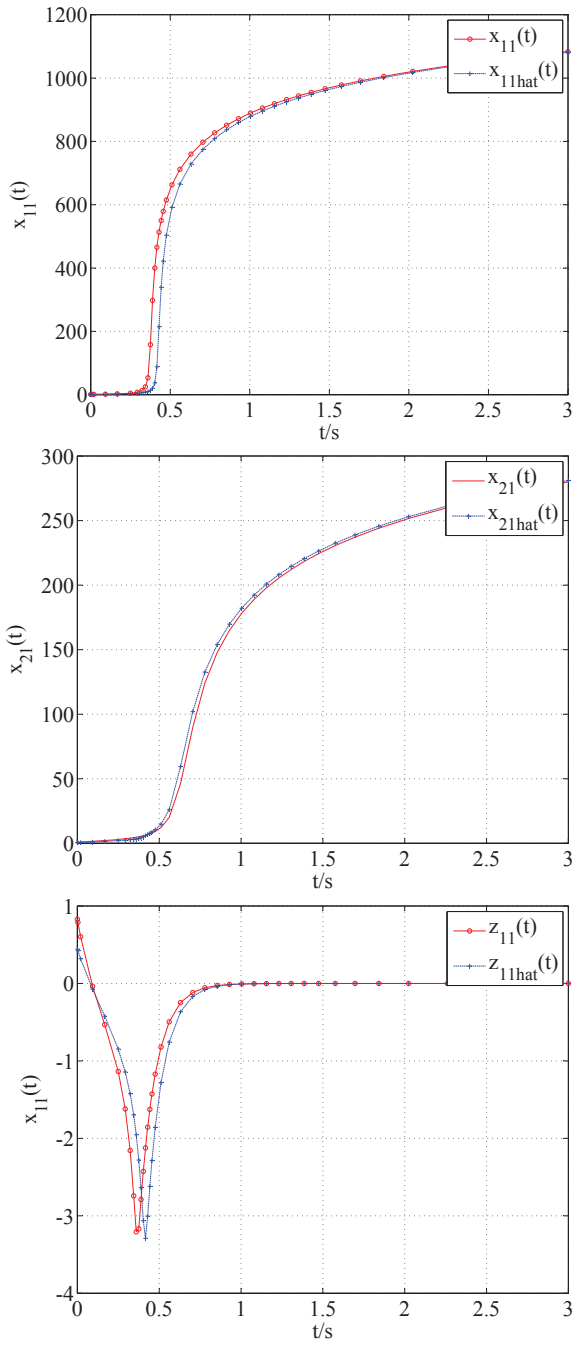

Fig. 5. State trajectories of the original states and their estimates of the first node system.
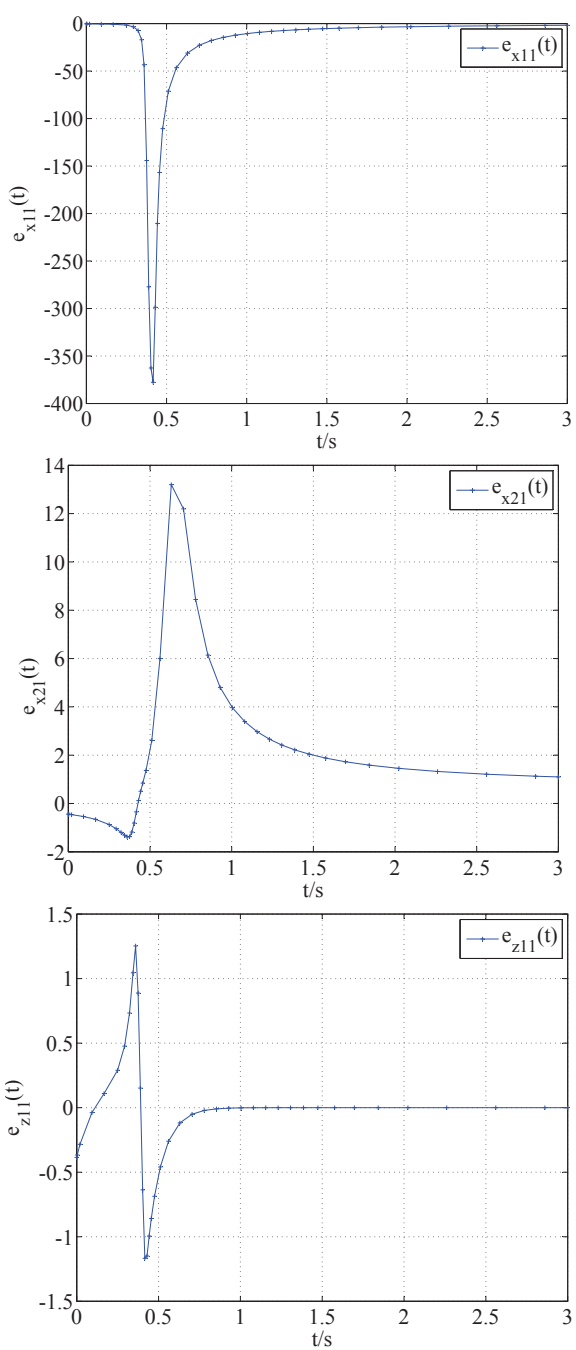

Fig. 6. Estimate error of the states of the first node system. 